Article

\title{
Impact of Dropwise Condensation on the Biomass Production Rate in Covered Raceway Ponds
}

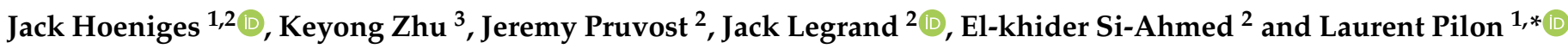 \\ 1 Mechanical and Aerospace Engineering Department, Henry Samueli School of Engineering and Applied \\ Science, University of California, Los Angeles, CA 90095, USA; jhoeniges@g.ucla.edu \\ 2 Université de Nantes, Oniris, CNRS, GEPEA, UMR 6144, F-44600 Saint-Nazaire, France; \\ jeremy.pruvost@univ-nantes.fr (J.P.); jack.legrand@univ-nantes.fr (J.L.); \\ el-khider.si-ahmed@univ-nantes.fr (E.-k.S.-A.) \\ 3 School of Aeronautical Science and Engineering, Beihang University, Beijing 100191, China; \\ zhukeyong@buaa.edu.cn \\ * Correspondence: pilon@seas.ucla.edu
}

Citation: Hoeniges, J.; Zhu, K.;

Pruvost, J.; Legrand, J; Si-Ahmed, E.-k.; Pilon, L. Impact of Dropwise Condensation on the Biomass Production Rate in Covered Raceway Ponds. Energies 2021, 14, 268. https://doi.org/10.3390/en14020268

Received: 2 December 2020 Accepted: 22 December 2020 Published: 6 January 2021

Publisher's Note: MDPI stays neutral with regard to jurisdictional clai$\mathrm{ms}$ in published maps and institutional affiliations.

Copyright: (C) 2021 by the authors. Licensee MDPI, Basel, Switzerland. This article is an open access article distributed under the terms and conditions of the Creative Commons Attribution (CC BY) license (https:// creativecommons.org/licenses/by/ $4.0 /)$.

\begin{abstract}
This study investigates the effect of condensed water droplets on the areal biomass productivity of outdoor culture systems with a free surface, protected by a transparent window or cover to prevent contamination and to control the growth conditions. Under solar radiation, evaporation from the culture causes droplets to condense on the interior surface of the cover. To quantify the effect of droplets on the system's performance, the bidirectional transmittance of a droplet-covered window was predicted using the Monte Carlo ray-tracing method. It was combined with a growth kinetics model of Chlorella vulgaris to predict the temporal evolution of the biomass concentration on 21 June and 23 September in Los Angeles, CA. A droplet contact angle of $30^{\circ}$ or $90^{\circ}$ and a surface area coverage of $50 \%$ or $90 \%$ were considered. Light scattering by the condensed droplets changed the direction of the incident sunlight while reducing the amount of light reaching the culture by up to $37 \%$. The combined effect decreased the daily areal biomass productivity with increasing droplet contact angle and surface area coverage by as much as $18 \%$. Furthermore, the areal biomass productivity of the system was found to scale with the ratio $X_{0} / a$ of the initial biomass concentration $X_{0}$ and the specific illuminated area $a$, as previously established for different photobioreactor geometries, but even in the presence of droplets. Finally, for a given day of the year, the optical thickness of the culture that yielded the maximum productivity was independent of the window condition. Thus, the design and operation of such a system should focus on maintaining a small droplet contact angle and surface area coverage and an optimum optical thickness to maximize productivity.
\end{abstract}

Keywords: light transfer; photobioreactor; biofuel; bioprocess engineering; design; modeling

\section{Introduction}

Biofuels derived from microalgae have the potential to serve as a renewable and carbon-neutral alternative to conventional transportation fuels [1]. Furthermore, microalgae have garnered interest as a source of food and dietary supplements that use significantly less resources than conventional agriculture due to their rapid growth and high nutrient and protein content [2]. Cost-effective production of algal biomass is essential to produce economically viable algae-based products. While microalgae grow readily in a variety of conditions, optimizing their growth poses various technical challenges [3]. Cultivation systems must be optimized to deliver light and nutrients to the microalgae while maintaining optimum growth conditions such as temperature, $\mathrm{pH}$, and dissolved $\mathrm{O}_{2}$ and balancing costs associated with construction, operation, land use, water use, and nutrient sourcing [4].

Microalgae cultivation systems are generally classified as open or closed. Open systems such as raceway ponds are inexpensive to build and operate and have been widely 
used for large-scale microalgae cultivation [4]. However, they can be easily contaminated; they lack effective temperature control; they suffer from water loss by evaporation; and they have relatively high harvesting costs due to their low cell concentration compared to closed systems [3]. Their operation also results in $\mathrm{CO}_{2}$ degassing [5]. By contrast, closed cultivation systems, also referred to as photobioreactors (PBRs), typically feature a lower risk of contamination and higher biomass productivity than open systems [3,6]. However, PBRs are typically more costly, and their scale up remains challenging [3]. Specifically, in flat horizontal or inclined culture systems, a transparent window covering the culture and containing an over-pressurized gas space can be used to (i) limit culture contamination, (ii) reduce $\mathrm{CO}_{2}$ degassing [5], and (iii) improve the control of the growth conditions [3]. Such an outdoor PBR consisting of a race pond with a paddle wheel sealed by a transparent plastic cover is illustrated in Figure 1a. The use of a transparent window enables the cultivation of a wider variety of species and thus greater potential for the production of high value-added products without significantly increasing construction and maintenance costs [3,6]. Regardless of the specifics of the cultivation system, water evaporation occurs when an outdoor microalgae culture is exposed to sunlight. When the culture is covered by a transparent window, evaporation leads to the formation of water droplets on the interior surface of the PBR cover, as illustrated in Figure 1b. However, the presence of droplets on the back side of a window has been shown to reduce its transmittance and change the direction of the transmitted light $[7,8]$. Both of these phenomena could impact the PBR biomass productivity [4,9]. However, to the best of our knowledge, the impact of droplet-covered windows on PBR performance has not been investigated to date.
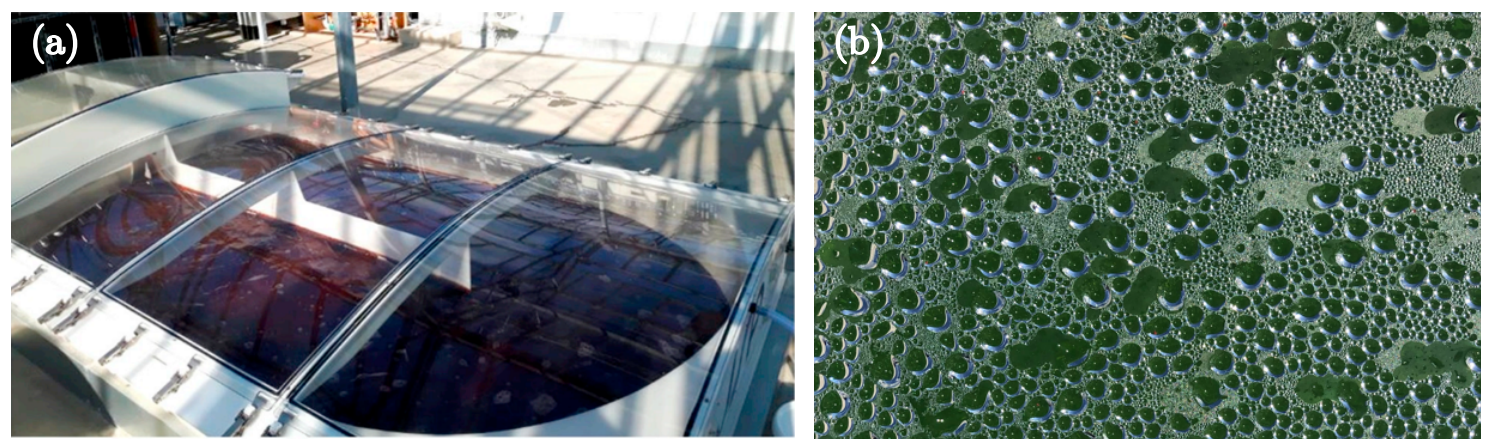

Figure 1. Photographs of (a) an outdoor photobioreactor at the R\&D facility AlgoSolis (Saint-Nazaire, France) with a clear window cover and (b) condensed droplets formed on the back side of a photobioreactor (PBR) window during a summer day.

The objective of this study is to quantify the effect of pendant droplets on the cover of outdoor microalgae cultivation systems on biomass productivity. To do so, light transfer was simulated through the PBR cover supporting pendant condensate droplets. The predicted bidirectional transmittance served as an input into combined light transfer and growth kinetics models in the culture of Chlorella vulgaris. Particular attention was paid to the effect of droplet contact angle, surface area coverage, culture depth, initial biomass concentration, and day of the year on the daily biomass production rate.

\section{Background}

\subsection{Light Transfer through Droplet-Covered PBR Windows}

The interaction between light and droplet-covered windows has been studied both analytically $[7,8,10-12]$ and experimentally [11,13-17]. Zhu et al. [8] simulated visible light transfer through a transparent window covered with non-absorbing cap-shaped water droplets on its back side. Light transfer was modeled using the Monte Carlo ray-tracing method for a wide range of droplet contact angle $\theta_{c}$ and surface area coverage $f_{A}$. Interestingly, both the normal-hemispherical transmittance $T_{n h}$ and the directional-hemispherical transmittance $T_{d h}$ of the droplet-covered window were found to be independent of droplet 
size distribution and droplet spatial arrangement. Similar results were observed for the bidirectional transmittance $T_{b d}$ [7]. The normal-hemispherical transmittance $T_{n h}$ was nearly independent of contact angle $\theta_{c}$ until it reached the critical angle $\theta_{c r}$ for total internal reflection at the droplet/air interface, i.e., for $\theta_{c}<\theta_{c r}$. However, for $\theta_{c}>\theta_{c r}$, the normal-hemispherical $T_{n h}$ and directional-hemispherical transmittance $T_{d h}$ decreased monotonically with increasing droplet surface area coverage $f_{A}$ and/or increasing contact angle $\theta_{c}$ until reaching a minimum at $\theta_{c}=90^{\circ}$. Similar results were found experimentally by previous studies $[11,17]$.

Zhu and Pilon [18] investigated the effect of absorption by the window and/or droplets on the transmittance of a window covered with cap-shaped droplets on its back side. In the case of a weakly absorbing window or droplets with absorption indices of $k_{w}=10^{-6}$ and $k_{d}=10^{-4}$, respectively, the normal-hemispherical transmittance $T_{n h}$ was observed to decrease only slightly. Furthermore, the dependence on droplet contact angle $\theta_{c}$ was the same as that for a non-absorbing window and droplets. Thus, in the case of water on plexiglass, whose absorption indices are less than $1.6 \times 10^{-7}$ and $1.8 \times 10^{-6}$, respectively, over much of the photosynthetically active radiation (PAR) region from 400 to $700 \mathrm{~nm}$, absorption by the window and droplets can be neglected $[19,20]$.

\subsection{Light Transfer in Microalgae Culture}

Light transfer through a well-mixed suspension of microalgae is governed by the radiative transfer equation (RTE) for a homogeneous absorbing, scattering, and nonemitting medium. The two-flux approximation can be used as an analytical solution to the one-dimensional RTE for flat plate PBRs or raceway ponds [21,22]. This approximation has been used extensively [21-25] and has been successfully validated against results from a 3D RTE solver for ponds and flat plate PBRs [22].

Souliès et al. [9] theoretically and experimentally investigated the effect of incidence angle on the growth of Chlorella vulgaris in thin flat panel PBRs artificially illuminated by collimated visible light with an incidence angle $\theta_{i}$ of $0^{\circ}$ or $60^{\circ}$. The intensity of the light source was adjusted such that the PBRs received the same radiative flux for both incidence angles. Nonetheless, the experimentally measured volumetric biomass productivity was observed to decrease from $0.029 \mathrm{~kg} \mathrm{~m}^{-3} \mathrm{~h}^{-1}$ for the PBR under normal incidence to $0.026 \mathrm{~kg} \mathrm{~m}^{-3} \mathrm{~h}^{-1}$ for the PBR under oblique incidence at $\theta_{i}=60^{\circ}$. Compared to normal incidence, light propagating through the culture at an oblique angle had a longer pathlength to reach a given depth. This caused light to be fully absorbed at shallower depths and prevented it from penetrating deeper into the PBR. Thus, a smaller fraction of the culture volume was illuminated, and the volumetric PBR biomass productivity decreased.

Pruvost et al. [25] theoretically investigated the maximum achievable productivity for outdoor PBRs cultivating Arthrospira platensis. Two scenarios were considered. The first was an idealized tracking PBR wherein the solar flux was normally incident throughout the day. The second was a fixed horizontal PBR that experienced oblique incidence during the day. Here, the oblique incidence was observed to decrease (i) the photon flux density incident on the PBR and (ii) the penetration depth of sunlight into the PBR culture, as demonstrated by Souliès et al. [9]. Together, these two effects resulted in a $31 \%$ decrease in the areal biomass productivity from 55 tons ha ${ }^{-1}$ year $^{-1}$ to 38 tons ha $^{-1}$ year $^{-1}$ for the "ideal" tracking PBR and the PBR under oblique incidence, respectively.

\subsection{Microalgae Growth Kinetics}

The time rate of change of the biomass concentration $X(t)$ in a PBR operated in batch mode can be modeled as [26]:

$$
\frac{d X}{d t}=\bar{\mu}(t) X(t)
$$

where $\bar{\mu}(t)$ is the average specific growth rate (in $\mathrm{h}^{-1}$ ) at time $t$. Various models have been proposed to predict the average specific growth rate $\bar{\mu}$ of microalgae as a function of the local spectral fluence rate $G_{\lambda}(z)$. Takache et al. [27] developed a growth kinetics 
model based on an energetic analysis of the excitation energy transfer in the photosynthetic apparatus. This model accounted for light limitation, photoinhibition, and cell respiration activity as a function of the local fluence rate [27]. Souliès et al. [9] further developed this model to account for the cell respiration activity based on the local rate of photon absorption (LRPA).

Lee et al. [22] studied the effect of PBR geometry on its daily biomass productivity. Microalgae growth in outdoor open ponds, vertical flat plate PBRs, and tubular PBRs cultivating Chlamydomonas reinhardtii was modeled accounting for light saturation and photoinhibition using the Haldane model [28]. The biomass loss rate from cell respiration was assumed to be constant [22,29]. Remarkably, for all PBR configurations, the biomass productivity was found to scale with the ratio of the initial biomass concentration $X_{0}$ and the specific illuminated area $a$ (in $\mathrm{m}^{-1}$ ) given by $a=S / V$ where $S$ is the illuminated surface area and $V$ is the culture volume [22,30]. In addition, the maximum PBR biomass productivity occurred at the same value of $X_{0} / a$ for all PBR geometries. Similar results were obtained from both experimental and theoretical data reported in previous studies $[31,32]$ despite the use of different microalgae species and growth models.

The present study aims to quantify the negative effect of condensed droplets on the window of outdoor covered raceway ponds on the time-dependent LRPA, biomass concentration, growth rate, and daily biomass productivity. This was achieved by coupling simulations of light transfer through droplet-covered windows with a simplified model for light transfer through the microalgae culture and a growth kinetics model for various solar conditions, droplet contact angle, and surface area coverage.

\section{Methods}

\subsection{Problem Statement}

Let us consider an outdoor raceway pond located in Los Angeles, CA, USA $\left(34.07^{\circ} \mathrm{N}\right.$, $118.44^{\circ} \mathrm{W}$ ) exposed to collimated solar radiation $G_{S, \lambda}$ on the summer solstice (21 June) and on the autumn equinox (23 September). The PBR was covered by a transparent horizontal window of thickness $H$ and refractive index $n_{w}$, as illustrated in Figure 2. The solar zenith angle $\theta_{z}$ was defined with respect to the outward pointing normal vector of the window surface, and the solar azimuth angle $\gamma_{s}$ was defined with respect to the due south direction where $\gamma_{s}=90^{\circ}$ corresponds to due west and $\gamma_{s}=-90^{\circ}$ corresponds to due east. Figure 2a shows the situation when the PBR window was dry and droplet-free. Figure $2 b$ schematically illustrates the PBR window partially covered by condensed droplets on its back side with surface area coverage $f_{A}$. The droplets were non-absorbing and capshaped with refractive index $n_{d}$, diameter $d$, projected diameter $d_{p}$, and contact angle $\theta_{c}$. The incident radiation was either back-scattered or transmitted through the dry or through the droplet-covered window into the PBR culture undergoing reflection or refraction at each interface. The PBR contained a well-mixed culture of depth $L$ and effective refractive index $n_{m}$ growing Chlorella vulgaris with time-dependent biomass concentration $X(t)$ (in $\mathrm{g} \mathrm{L}^{-1}$ ) and spectral average mass absorption $\bar{A}_{a b s, \lambda}$ and scattering $\bar{S}_{s c a, \lambda}$ cross-sections (in $\left.\mathrm{m}^{2} \mathrm{~kg}^{-1}\right)$, and spectral scattering phase function $\Phi_{\lambda}(\Theta)$. The culture depth $L$ varied from $0.1 \mathrm{~m}$ to $0.3 \mathrm{~m}$, and the initial biomass concentration $X_{0}$ varied from $0.01 \mathrm{~g} \mathrm{~L}^{-1}$ to $1 \mathrm{~g} \mathrm{~L}^{-1}$. 
(a) Dry window

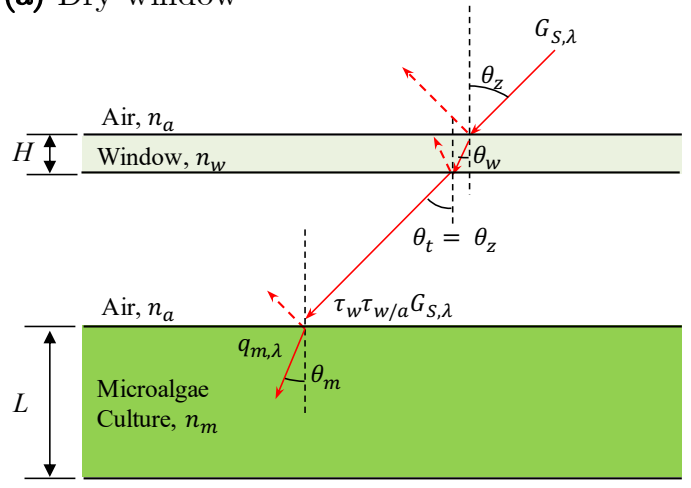

(b) Droplet-covered window

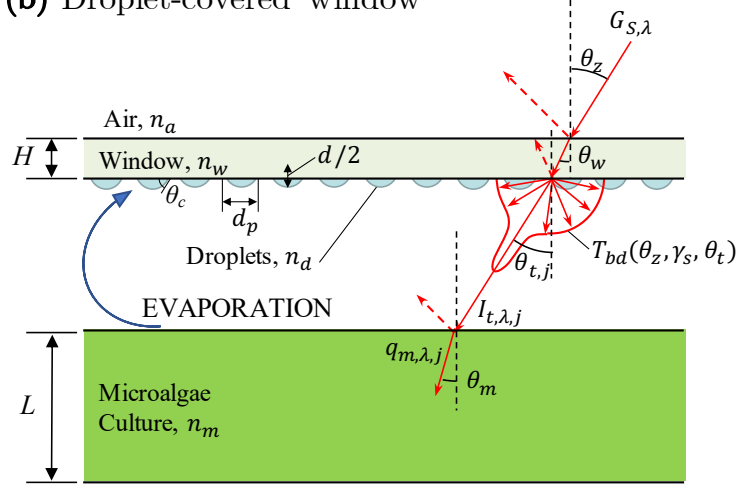

Figure 2. Side view (not to scale) of the covered outdoor PBRs studied with (a) a dry window and (b) a droplet-covered window. In both cases, a portion of the solar radiation was reflected at each interface before the radiative flux $q_{m, \lambda, j}$ was transmitted into the microalgae culture at angle $\theta_{m}$. Scattering by cap-shaped droplets on the window's back side was described by the bidirectional transmittance $T_{b d}$.

\subsection{Assumptions}

Light transfer through the droplet-covered windows was simulated using the Monte Carlo ray-tracing method [7,8,12]. To do so, the following assumptions were made [8]: (1) all interfaces were considered optically smooth. In other words, surface roughness was considered to be much less than the wavelength of light in the photosynthetically active region $(\lambda=400-700 \mathrm{~nm})$ such that reflection and transmission were specular and Snell's law and Fresnel's equations were valid. (2) The dimensions of the droplets and windows were much larger than the wavelength of light such that geometric optics prevailed. (3) Both the window and the droplets were considered to be non-absorbing over the PAR region. (4) The droplets were considered to be cap-shaped with a constant curvature. (5) The droplets were monodisperse with an arbitrary diameter $d=100 \mu \mathrm{m}$ since the bidirectional transmittance of transparent windows with non-absorbing droplets was found to be independent of the droplet size $[7,8]$. (6) The windows simulated had a surface area coverage $f_{A}$ of $50 \%$ or $90 \%$. Although local conditions may impact the amount of condensate present on the window, the range of $f_{A}$ was selected to represent a moderate and a worst-case-scenario droplet coverage. (7) Droplet contact angle $\theta_{c}$ was $30^{\circ}$ or $90^{\circ}$. These two angles were chosen to approximately represent water on glass and water on a hydrophobic cover, respectively. (8) The refractive index of the air, window, water droplets, and microalgae culture were taken as constant across the PAR region and equal to $n_{a}=1.0, n_{w}=1.5, n_{d}=1.33$, and $n_{m}=1.33$, respectively. Thus, light scattering by the droplets was independent of wavelength. (9) The diffuse part of the incident sunlight was neglected such that all solar radiation incident upon the PBR window was collimated, and its direction $\left(\theta_{s}, \gamma_{s}\right)$ varied during the course of the day.

Predictions of light transfer and microalgae growth in the outdoor PBR were based on the assumptions that (10) the PBR was operated in the light-limited regime wherein growth was only a function of the amount of light available to the microalgae $[9,22,25]$. Thus, the PBR was not limited by any other operating parameters such as temperature, $\mathrm{pH}$, and nutrient availability, and changes in PBR biomass productivity could be attributed to light scattering by the droplets. (11) The microalgae culture was well-mixed with uniform biomass concentration $X(t)$ throughout the PBR. (12) The cells were randomly oriented. (13) The liquid medium was non-scattering and non-absorbing over the PAR region. (14) The radiation characteristics of Chlorella vulgaris were constant throughout the day and taken from the literature [9]. (15) All walls of the PBR were considered non-reflective and perfectly absorbing. (16) Edge effects and shading from the side walls were considered negligible so that light transfer in the PBR culture could be treated as one-dimensional and depended only on the culture depth $z$. 


\subsection{Light Transfer through Droplet-Covered PBR Windows}

For PBRs covered by dry windows, Figure 2a illustrates that the incoming collimated spectral solar irradiance $G_{S, \lambda}\left(\right.$ in $\mu \mathrm{mol}_{h v} \mathrm{~m}^{-2} \mathrm{~s}^{-1}$ ) was reflected and refracted at the air/window interface, at the window/air interface, and at the air/microalgae culture interface before being transmitted into the PBR culture. The transmissivity at the air/window and the air/microalgae culture interface, denoted by $\tau_{a / w}$ and $\tau_{a / m}$, respectively, was determined from Fresnel's relation given by [33]:

$$
\tau_{1 / 2}=1-\frac{1}{2}\left[\frac{\tan ^{2}\left(\theta_{1}-\theta_{2}\right)}{\tan ^{2}\left(\theta_{1}+\theta_{2}\right)}+\frac{\sin ^{2}\left(\theta_{1}-\theta_{2}\right)}{\sin ^{2}\left(\theta_{1}+\theta_{2}\right)}\right]
$$

where $\theta_{1}$ and $\theta_{2}$ are the incident and refracted angles across the Medium 1/Medium 2 interface given by Snell's law $n_{1} \sin \theta_{1}=n_{2} \sin \theta_{2}$. Then, the spectral radiative flux at the upper boundary of the microalgae culture $q_{m, \lambda}$ (Figure 2a) was expressed as a function of the solar position $\left(\theta_{z}, \gamma_{s}\right)$ and the transmissivity at each interface as:

$$
q_{m, \lambda}\left(\theta_{z}, \gamma_{s}\right)=\tau_{w} \tau_{a / m} G_{S, \lambda}\left(\theta_{z}, \gamma_{s}\right) \cos \theta_{z} .
$$

where $\tau_{w}$ is the transmissivity of the window given by $\tau_{w}=\tau_{a / w} /\left(2-\tau_{a / w}\right)$ [34].

For droplet-covered windows, the incident sunlight was scattered into the transmitted direction described by the polar $\theta_{t}$ and azimuthal $\varphi_{t}$ transmission angles (Figure $2 \mathrm{~b}$ ). The resulting angular distribution of the transmitted light was described by the bidirectional transmittance $T_{b d}$ in the transmitted direction $\left(\theta_{t}, \varphi_{t}\right)$ defined as [33,34]:

$$
T_{b d}\left(\theta_{z}, \gamma_{s}, \theta_{t}, \varphi_{t}\right)=\frac{I_{t, \lambda}\left(\theta_{z}, \gamma_{s}, \theta_{t}, \varphi_{t}\right)}{G_{S, \lambda}\left(\theta_{z}, \gamma_{s}\right) \cos \theta_{z}}
$$

where $I_{t, \lambda}$ is the spectral radiative intensity transmitted in the direction $\left(\theta_{t}, \varphi_{t}\right)$. Note that $T_{b d}$ is wavelength independent since the optical properties of the window and droplets were assumed to be constant over the PAR region.

In order to be coupled with the one-dimensional light transfer analysis in the microalgae culture, the bidirectional transmittance $T_{b d}\left(\theta_{z}, \gamma_{s}, \theta_{t}, \varphi_{t}\right)$ was averaged over the transmitted azimuthal angle $\varphi_{t}$ to yield the one-dimensional bidirectional transmittance $\bar{T}_{b d}\left(\theta_{z}, \gamma_{s}, \theta_{t}\right)$ according to [7]:

$$
\bar{T}_{b d}\left(\theta_{z}, \gamma_{s}, \theta_{t}\right)=\frac{1}{2 \pi} \int_{0}^{2 \pi} T_{b d}\left(\theta_{z}, \gamma_{s}, \theta_{t}, \varphi_{t}\right) \mathrm{d} \varphi_{t} .
$$

Then, $\bar{T}_{b d}\left(\theta_{z}, \gamma_{s}, \theta_{t}\right)$ (in sr ${ }^{-1}$ ) gave the average intensity transmitted in direction $\theta_{t}$ due to solar radiation from the incident direction $\left(\theta_{z}, \gamma_{s}\right)$. The one-dimensional bidirectional transmittance $\bar{T}_{b d}\left(\theta_{z}, \gamma_{s}, \theta_{t}\right)$ was then used to express the transmitted intensity $I_{t, \lambda}$ through the droplet-covered window into direction $\theta_{t}$ as:

$$
I_{t, \lambda}\left(\theta_{z}, \gamma_{s}, \theta_{t}\right)=G_{S, \lambda}\left(\theta_{z}, \gamma_{s}\right) \cos \theta_{z} \bar{T}_{b d}\left(\theta_{z}, \gamma_{s}, \theta_{t}\right)
$$

\subsection{Light Transfer in Microalgae Culture}

For wet windows, droplets scattered the incident sunlight into different directions in the transmission hemisphere. The latter can be discretized into $M$ directions $\left(\theta_{t, j}\right)_{1 \leq j \leq M}$. The spectral radiative flux $q_{m, \lambda, j}$ reaching the upper boundary of the microalgae culture in the $\theta_{t, j}$ direction can be written as:

$$
q_{m, \lambda, j}\left(\theta_{z}, \gamma_{s}, \theta_{t, j}\right)=\tau_{a / m} I_{t, \lambda, j} \cos \theta_{t, j} \Delta \Omega_{t, j}
$$

where the solid angle is given by $\Delta \Omega_{t, j}=2 \pi \sin \theta_{t, j} \Delta \theta_{t, j}$. Due to refraction, radiation transmitted through the window at angle $\theta_{t, j}$ was then transmitted through the air/microalgae 
culture interface at an angle $\theta_{m, j}=\sin ^{-1}\left(n_{a} \sin \theta_{t, j} / n_{m}\right)$. Then, according to the two-flux approximation, the resulting local fluence rate $G_{\lambda, j}(z)$ at depth $z$ within the culture due to the spectral radiative flux $q_{m, \lambda, j}$ incident at angle $\theta_{t, j}$ on the upper boundary of the culture was expressed as [21]:

$$
\frac{G_{\lambda, j}\left(z, \theta_{t, j}\right)}{q_{m, \lambda, j}\left(\theta_{t, j}\right)}=\frac{2}{\cos \theta_{m, j}} \frac{\left(1+\alpha_{\lambda}\right) e^{\delta_{\lambda, j}(L-z)}-\left(1-\alpha_{\lambda}\right) e^{-\delta_{\lambda, j}(L-z)}}{\left(1+\alpha_{\lambda}\right)^{2} e^{\delta_{\lambda, j} L}-\left(1-\alpha_{\lambda}\right)^{2} e^{-\delta_{\lambda, j} L}}
$$

where $\alpha_{\lambda}$ and $\delta_{\lambda, j}$ are given by [21]:

$$
\alpha_{\lambda}=\sqrt{\frac{\bar{A}_{a b s, \lambda}}{\bar{A}_{a b s, \lambda}+2 b_{\lambda} \bar{S}_{s c a, \lambda}}} \quad \text { and } \quad \delta_{\lambda, j}=\frac{\alpha_{\lambda} X}{\cos \theta_{m, j}}\left(\bar{A}_{a b s, \lambda}+2 b_{\lambda} \bar{S}_{s c a, \lambda}\right) .
$$

here, the average spectral mass absorption $\bar{A}_{a b s, \lambda}$ and scattering $\bar{S}_{s c a, \lambda}$ cross-sections in $\mathrm{m}^{2} \mathrm{~kg}^{-1}$ (shown in Figure A1) are the properties of the microalgae species being cultivated and were taken from [9] for a cell mean radius of $2 \mu \mathrm{m}$ and chlorophyll $a$, chlorophyll $b$, and photoprotective carotenoid (PPC) concentrations of $3.3 \mathrm{wt} . \%, 0.85 \mathrm{wt} . \%$, and $0.85 \mathrm{wt} . \%$, respectively. Assuming axisymmetric scattering by the culture, the backward scattering ratio $b_{\lambda}$ is defined as [21]:

$$
b_{\lambda}=\frac{1}{2} \int_{\pi}^{\pi / 2} \Phi_{\lambda}(\Theta) \sin \Theta \mathrm{d} \Theta .
$$

here, $\Phi_{\lambda}(\Theta)$ is the so-called scattering phase function representing the probability that photons in the solid angle $\mathrm{d} \Omega_{i}$ about direction $\hat{s}_{i}$ are scattered into the solid angle $\mathrm{d} \Omega$ about direction $\hat{s}$ with $\Theta$ defined as the angle between the scattered direction $\hat{s}$ and the incident direction $\hat{s}_{i}$. The scattering phase function was predicted on a spectral basis for Chlorella vulgaris using the Lorenz-Mie theory for a coated sphere according to the method described in [35]. The resulting spectral backward scattering ratio $b_{\lambda}$ is shown in Figure A1 of Appendix A.

For PBRs with droplet-covered windows, the solar radiation was transmitted through the window into many directions as depicted in Figure $2 b$. Thus, for a given solar position $\left(\theta_{z}, \gamma_{s}\right)$, the spectral local fluence rate $G_{\lambda}(z)$ inside the culture covered by a droplet-covered window was found by summing the contribution from all transmission directions $\theta_{t, j}$ according to:

$$
G_{\lambda}(z)=\sum_{j=1}^{M+1} G_{\lambda, j}\left(z, \theta_{t, j}\right)
$$

For the dry window PBR, the light reaching the upper boundary of the microalgae culture was collimated, and $G_{\lambda}(z)$ is given by Equation (8) for that specific direction.

The ability of the microalgae to absorb the local spectral fluence rate $G_{\lambda}(z)$ was then described by the local rate of photon absorption (LRPA) $\mathcal{A}(z)\left(\right.$ in $\left.\mu \mathrm{mol}_{h v} \mathrm{~kg}^{-1} \mathrm{~s}^{-1}\right)$ defined as [9]:

$$
\mathcal{A}(z)=\int_{P A R} \bar{A}_{a b s, \lambda} G_{\lambda}(z) d \lambda .
$$

\subsection{Microalgae Growth Kinetics}

The microalgae growth kinetic model proposed by Takache et al. [27] and further developed by Souliès et al. [9] for Chlorella vulgaris was employed to predict the average growth rate $\bar{\mu}$ of the microalgae culture. As discussed previously, this model accounted for light limitation, photoinhibition, and cell respiration activity as a function of the LRPA $\mathcal{A}(z)$ [27]. First, the local specific rate of oxygen production or consumption $J_{\mathrm{O}_{2}}(z)\left(\right.$ in $\left.\mathrm{mol}_{\mathrm{O}_{2}} \mathrm{~kg}_{\mathrm{X}}^{-1} \mathrm{~s}^{-1}\right)$ was calculated according to [9]:

$$
J_{\mathrm{O}_{2}}(z)=\left[\rho_{M} \frac{K}{K+\mathcal{A}(z)} \bar{\phi}_{\mathrm{O}_{2}}^{\prime} \mathcal{A}(z)-\frac{J_{\mathrm{NADH}_{2}}}{v_{\mathrm{NADH}_{2}-\mathrm{O}_{2}}} \frac{K_{r}}{K_{r}+\mathcal{A}(z)}\right] .
$$


here, $\rho_{M}$ is the maximum energy yield for photon conversion, $\bar{\phi}_{\mathrm{O}_{2}}^{\prime}\left(\right.$ in $\left.\operatorname{mol}_{\mathrm{O}_{2}} \mu \mathrm{mol}_{h v}^{-1}\right)$ is the mole quantum yield of $\mathrm{O}_{2}$ for the Z-scheme of photosynthesis, $K$ (in $\mu \mathrm{mol}_{h v} \mathrm{~kg}^{-1} \mathrm{~s}^{-1}$ ) is the half-saturation constant for photosynthesis, $J_{\mathrm{NADH}_{2}}\left(\right.$ in $\mathrm{mol}_{\mathrm{NADH}_{2}} \mathrm{~kg}_{\mathrm{X}}^{-1} \mathrm{~s}^{-1}$ ) is the specific rate of cofactor regeneration on the respiratory chain, related to the oxygen consumption by the stoichiometric coefficient of cofactor regeneration on the respiratory chain $v_{\mathrm{NADH}_{2}-\mathrm{O}_{2}}$, and $K_{r}$ (in $\left.\mu \mathrm{mol}_{h v} \mathrm{~kg}^{-1} \mathrm{~s}^{-1}\right)$ is the half saturation constant describing the inhibition of respiration in light. These parameters were taken from [9] and are summarized in Table A1. These parameters and the absorption $\bar{A}_{a b s, \lambda}$ and scattering $\bar{S}_{s c a, \lambda}$ cross-sections corresponded to the same culture [9]. The average specific rate of oxygen production $\bar{J}_{O_{2}}$ over the depth $L$ of the PBR was defined as [9]:

$$
\bar{J}_{O_{2}}=\frac{1}{L} \int_{0}^{L} J_{O_{2}}(z) \mathrm{d} z \text {. }
$$

Then, $\bar{J}_{\mathrm{O}_{2}}$ was used to predict the average specific growth rate $\bar{\mu}\left(\right.$ in s $^{-1}$ ) via the stoichiometric relationship between the production of oxygen and the production of biomass [9]:

$$
\bar{\mu}=\frac{\bar{J}_{O_{2}} M_{x}}{v_{\mathrm{O}_{2}-X}}
$$

where $M_{x}$ (in $\mathrm{kg}_{x} \operatorname{mol}_{\mathrm{C}}^{-1}$ ) is the C-molar mass in the biomass given by $\mathrm{C}_{m} H_{p} \mathrm{O}_{n}$ and $v_{\mathrm{O}_{2}-\mathrm{X}}$ is the stoichiometric coefficient of the oxygen production.

Finally, the temporal evolution of the biomass concentration $X(t)$ was found by integrating Equation (1). Then, to assess the impact of droplets on PBR performance, the areal biomass productivity for a given day $P\left(\right.$ in $\mathrm{kg} \mathrm{m}^{-2}$ day $^{-1}$ ) was expressed as:

$$
P=\left(X_{f}-X_{0}\right) L
$$

where $X_{0}$ and $X_{f}$ are the initial and final biomass concentrations, respectively, given by $X_{0}=X\left(t_{0}=7 \mathrm{am}\right)$ and $X_{f}=X\left(t_{f}=7 \mathrm{pm}\right)$ on 21 June and $X_{0}=X\left(t_{0}=9 \mathrm{am}\right)$ and $X_{f}=X\left(t_{f}=5 \mathrm{pm}\right)$ on 23 September.

Another important PBR performance metric that can be used to assess the effect of droplets is the so-called illuminated fraction $\gamma$ given by [25]:

$$
\gamma=\frac{z_{c}}{L}
$$

where $z_{c}$ is the maximum culture depth at which the local LRPA $\mathcal{A}(z)$ still yields positive photosynthetic growth, i.e., $\mathcal{A}\left(z_{c}\right)=\mathcal{A}_{c}$ where $\mathcal{A}_{c}$ is the minimum LRPA required to prevent biomass loss due to respiration [9]. When $\gamma=1$, positive photosynthetic growth occurs throughout the entire culture, and all incident light available for photosynthesis is absorbed by the culture. Under these conditions, the maximum average growth rate $\bar{\mu}_{\text {max }}$ is reached for given light conditions [25]. When $\gamma<1$, dark zones are present in the culture volume, and biomass loss occurs due to respiration. By contrast, $\gamma>1$ indicates that excess light is available for photosynthesis.

\subsection{Initial and Boundary Conditions}

The solar spectrum incident upon the PBR was determined by the PBR location and the time of year. Table 1 shows the value of the solar zenith $\theta_{z}$ and azimuth $\gamma_{s}$ angles associated with each simulated time on 21 June and 23 September. Figure $3 a, b$ shows the spectral solar direct normal irradiance $G_{S, \lambda}\left(\right.$ in $\mu \mathrm{mol}_{h v} \mathrm{~m}^{-2} \mathrm{~s}^{-1}$ ) in Los Angeles predicted by the Simple Model of the Atmospheric Radiative Transfer of Sunshine (SMARTS) [36] for wavelengths in the PAR region from 400 to $700 \mathrm{~nm}$ between 7 a.m. and 7 p.m. on 21 June and 9 a.m. and 5 p.m. on 23 September, respectively. As mentioned previously, edge effects were neglected, and the bottom of the PBR was considered to be black. The initial biomass concentration $X_{0}=X\left(t_{0}\right)$ was varied between 0.01 and $1.0 \mathrm{~g} \mathrm{~L}^{-1}$. 
(a) June $21^{\text {st }}$

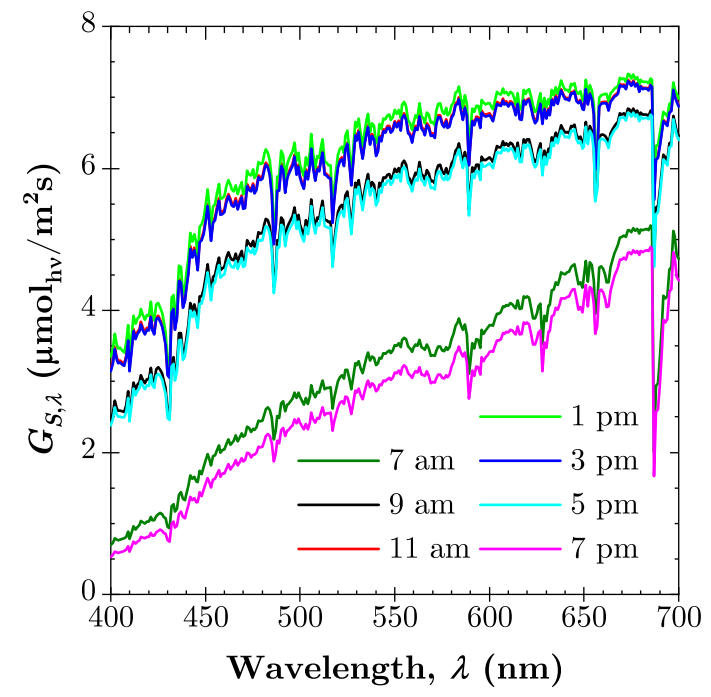

(b) September $23^{\text {rd }}$

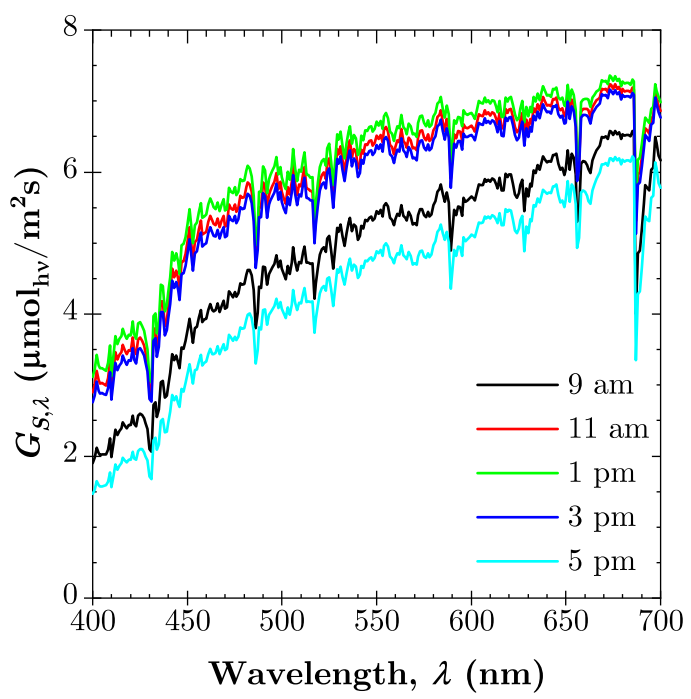

Figure 3. Incident collimated solar irradiance $G_{S, \lambda}$ over the PAR region at different times of day simulated on (a) 21 June and (b) 23 September in Los Angeles, CA.

Table 1. Solar zenith $\theta_{z}$ and azimuth $\gamma_{s}$ angles (in degrees) for the different times simulated on 21 June 21 and 23 September 23 in Los Angeles, CA.

\begin{tabular}{ccccc}
\hline Time & \multicolumn{2}{c}{ 21 June } & \multicolumn{2}{c}{ 23 September } \\
& $\boldsymbol{\theta}_{\boldsymbol{z}}$ & $\gamma_{\boldsymbol{s}}$ & $\boldsymbol{\theta}_{\boldsymbol{z}}$ & $\boldsymbol{\gamma}_{\boldsymbol{s}}$ \\
\hline 7 a.m. & 76.2 & -109.2 & - & - \\
9 a.m. & 52.0 & -94.7 & 62.9 & -69.6 \\
11 a.m. & 27.3 & -74.8 & 42.3 & -41.6 \\
1 p.m. & 10.7 & 5.5 & 34.4 & 6.2 \\
3 p.m. & 29.1 & 76.9 & 46.5 & 49.5 \\
5 p.m. & 53.8 & 95.8 & 68.4 & 74.2 \\
7 p.m. & 77.9 & 110.3 & - & - \\
\hline
\end{tabular}

\subsection{Method of Solution}

Figure 4 shows a block diagram of the procedure used to predict the final biomass concentration $X_{f}$ and the PBR daily areal biomass productivity $P$. The droplet parameters $\left(\theta_{c}, f_{A}\right)$, the spectral solar irradiance $G_{S, \lambda}$, and the solar incidence angles $\left(\theta_{z}, \gamma_{s}\right)$ at initial time $t=t_{0}$ were the input parameters for modeling light transfer through the PBR window. The appropriate light transfer model was then applied according to the window condition. Then, the resulting spectral radiative flux at the upper boundary of the microalgae culture $q_{m, \lambda}(t)$ and the PBR operating parameters $\left(X_{0}, L\right)$ were used to model light transfer and growth kinetics in the culture to predict the biomass concentration $X(t)$. This process was then repeated for subsequent time steps until the final biomass concentration $X_{f}=X\left(t_{f}\right)$ and the daily areal biomass productivity $P$ were computed. 


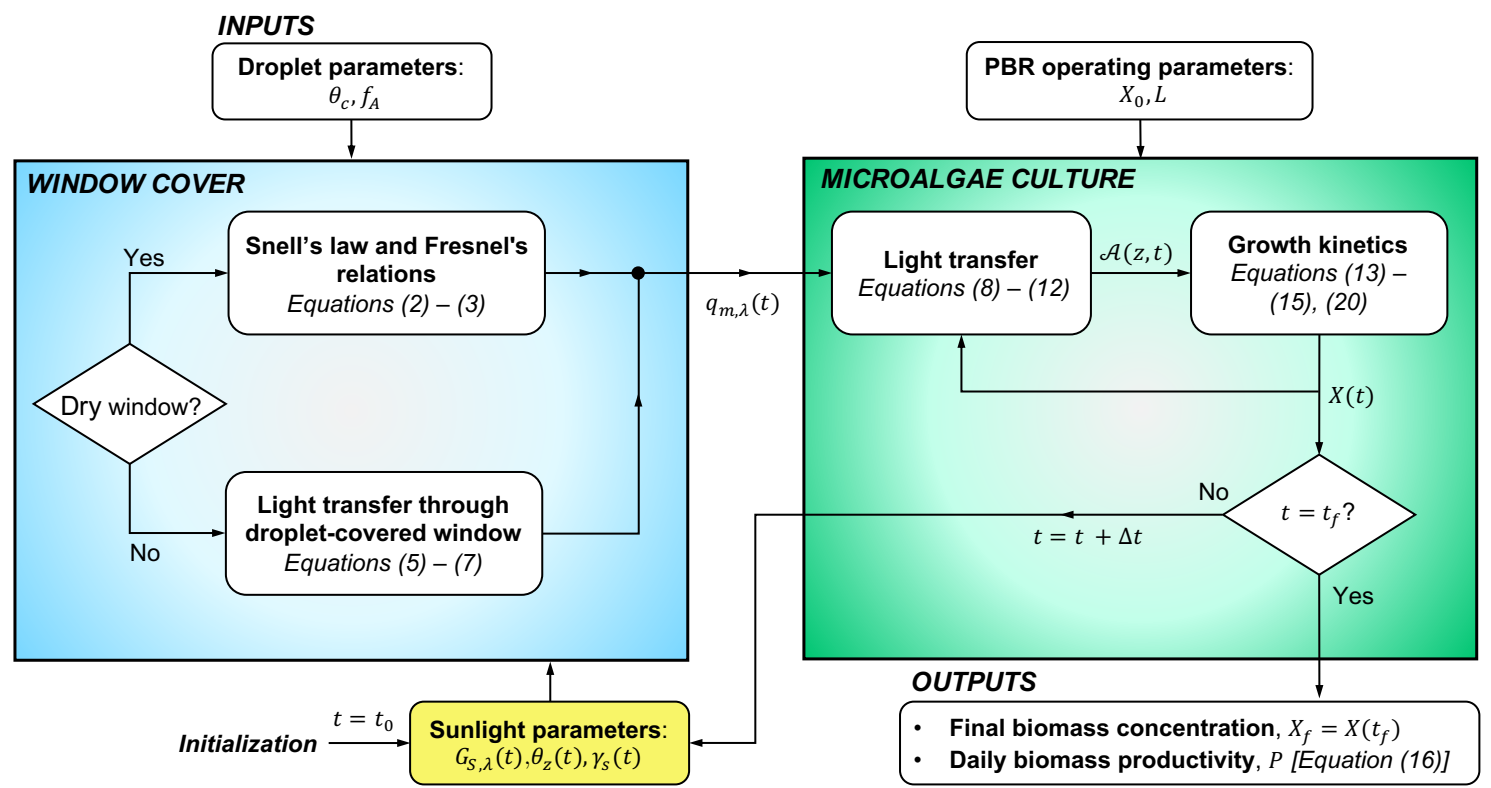

Figure 4. Block diagram of the procedure used to predict the final daily biomass $X_{f}$ and daily areal biomass productivity $P$ for PBRs with dry and droplet-covered windows.

Light transfer through the dry window and into the microalgae culture was modeled using Equations (2) and (3). For a droplet-covered window, the Monte Carlo ray-tracing method was used to predict the bidirectional transmittance $T_{b d}\left(\theta_{z}, \gamma_{s}, \theta_{t}, \gamma_{t}\right)$. Here, a large number of discrete collimated photon bundles or "rays" were launched in direction $\left(\theta_{z}, \gamma_{s}\right)$ towards the window covered by computationally-generated droplets. The method and algorithm were described in detail in $[7,8,12]$ and need not be repeated. In brief, the polar transmission angle $\theta_{t}$ ranged from $0^{\circ}$ to $90^{\circ}$ and was discretized into $M+1$ angular intervals where $M=90$, with $\Delta \theta_{t, j=1}=\Delta \theta_{t, j=M+1}=\pi / 4 M$ (in rad) for the first and last intervals at angles $\theta_{t, 1}=\pi / 8 M$ (in rad) and $\theta_{t, M+1}=\pi / 2-\pi / 8 M$ (in rad) while the interval $\left(\Delta \theta_{t, j}\right)_{2 \leq j \leq M}=\pi / 2 M$ (in rad) was used for all other transmission angles. The azimuthal transmission angle $\varphi_{t}$ ranged from $0^{\circ}$ to $360^{\circ}$ in uniform intervals of $\Delta \varphi_{t}=$ $\pi / 180$ (in rad). At each interface a ray encountered, the interface reflectivity was calculated using Fresnel's equations. Then, a random number selected from the uniform distribution was compared to the interface reflectivity to determine if the ray was reflected or refracted. The new ray direction was governed by specular reflection or Snell's law for refracted rays. The next interface the ray encountered was then determined using the geometry of the 3D simulation domain. For a non-absorbing window and droplets, this process continued until the ray was either (i) transmitted through the window and droplets or (ii) reflected away from the window. Then, the fraction of solar energy transmitted through the droplet-covered window for a given solar position $\left(\theta_{z}, \gamma_{s}\right)$ was represented by the directional-hemispherical transmittance $T_{d h}$ expressed as [8]:

$$
T_{d h}\left(\theta_{z}, \gamma_{s}\right)=\frac{N_{t}}{N_{i}}
$$

where $N_{i}$ is the total number of rays incident on the window and $N_{t}$ is the total number of transmitted rays. Furthermore, Equation (4) was recast to express the bidirectional transmittance for a given solar position $\left(\theta_{z}, \gamma_{s}\right)$ and transmitted direction $\left(\theta_{t, j}, \varphi_{t, k}\right)$ from the output of the Monte Carlo ray-tracing simulation according to [7]:

$$
T_{b d, j k}\left(\theta_{z}, \gamma_{s}, \theta_{t, j}, \varphi_{t, k}\right)=\frac{N_{t, j k}}{N_{i} \cos \theta_{t} \Delta \Omega_{t, j k}}
$$


where $N_{t, j k}$ is the number of rays transmitted into the solid angle $\Delta \Omega_{t, j k}=\sin \theta_{t, j} \Delta \theta_{t, j} \Delta \varphi_{t, k}$. In order to achieve numerical convergence for $T_{b d, j k}, N_{i}=10^{7}$ rays were simulated [7]. Then, the resulting spectral radiative flux at the upper boundary of microalgae culture $q_{m, \lambda, j}$ was calculated using the bidirectional transmittance $T_{b d, j k}$ and Equations (5)-(7).

Next, the LRPA $\mathcal{A}(z, t)$ accounting for all transmitted directions was predicted via the two-flux approximation according to Equations (8)-(12). Then, the growth kinetics model was used to predict the average specific growth rate $\bar{\mu}(t)$ based on Equations (13)-(15). The biomass concentration at time $t+\Delta t$ was predicted from the biomass concentration $X(t)$ at time $t$ by integrating Equation (1) according to [22]:

$$
X(t+\Delta t)=X(t)[1+\bar{\mu}(t) \Delta t] .
$$

Light transfer through the droplet-covered window was simulated in time increments of $\Delta t=2 \mathrm{~h}$ from 7 a.m. to $7 \mathrm{p} . \mathrm{m}$. on 21 June and 9 a.m. to 5 p.m. on 23 September. To check for numerical convergence, $X(t)$ and $\bar{\mu}(t)$ were predicted by simulating light transfer through a dry window PBR using a time increment of $\Delta t=0.5 \mathrm{~h}$ and $\Delta t=2 \mathrm{~h}$ (see Figure A2). The maximum relative errors throughout the day for the biomass $X(t)$ were less than $0.1 \%$ and $0.3 \%$ on 21 June and 23 September, respectively. Thus, a time increment of $2 \mathrm{~h}$ was considered to be an appropriate compromise between reasonable computation time and sufficient accuracy.

\section{Results and Discussion}

\subsection{Light Transfer through Droplet Covered PBR Windows}

Figure $5 \mathrm{a}, \mathrm{b}$ show the one-dimensional bidirectional transmittance $\bar{T}_{b d}\left(\theta_{z}, \gamma_{s}, \theta_{t}\right)$ as a function of the transmission angle $\theta_{t}$ at 1 p.m. and 5 p.m. for various window conditions on 21 June and 23 September, respectively. On 21 June at 1 p.m., all window conditions showed a strong peak in $\bar{T}_{b d}$ at the transmission angle equal to the solar zenith angle (i.e., $\theta_{t}=\theta_{z}$ ) with a large surrounding lobe. At $5 \mathrm{pm}$, as the solar zenith angle $\theta_{z}$ increased, the corresponding peak in $\bar{T}_{b d}$ decreased, and the surrounding lobes became wider for a given droplet configuration. This was indicative of increased scattering at larger solar zenith angles. Indeed, for a window with droplets of a contact angle of $\theta_{c}=90^{\circ}$ and surface area coverage $f_{A}=90 \%$ on 23 September at 5 p.m., so much scattering occurred that $\bar{T}_{b d}$ peaked around $\theta_{t}=45^{\circ}$ despite a solar zenith angle $\theta_{z}$ of $68^{\circ}$ at this time.

Figure $5 \mathrm{c}, \mathrm{d}$ show the temporal evolution of the average transmission angle $\bar{\theta}_{t}$ defined as the transmission angle $\theta_{t}$ weighted by the one-dimensional bidirectional transmittance $\bar{T}_{b d}\left(\theta_{z}, \gamma_{s}, \theta_{t}\right)$ for a given solar position $\left(\theta_{z}, \gamma_{s}\right)$, i.e.,

$$
\bar{\theta}_{t}=2 \pi \int_{0}^{\pi / 2} \bar{T}_{b d}\left(\theta_{z}, \gamma_{s}, \theta_{t}\right) \theta_{t} \sin \theta_{t} \mathrm{~d} \theta_{t} .
$$

In the case of a dry window, no scattering occurred, and the transmission angle was equal to the incident angle, i.e., $\theta_{t}=\theta_{z}$. However, for condensate-covered windows, at all times except between 11 a.m. and 3 p.m. on 21 June, light scattering by the droplets reduced the average transmission angle by as much as $27^{\circ}$ and $20^{\circ}$ on 21 June and 23 September, respectively. In other words, for large solar zenith angles $\theta_{z}$, the droplets reduced the transmittance, but scattered sunlight in directions closer to normal incidence, causing the transmitted light to penetrate deeper into the culture compared to a culture covered by a dry window. 
(a) June $21^{\text {st }}$

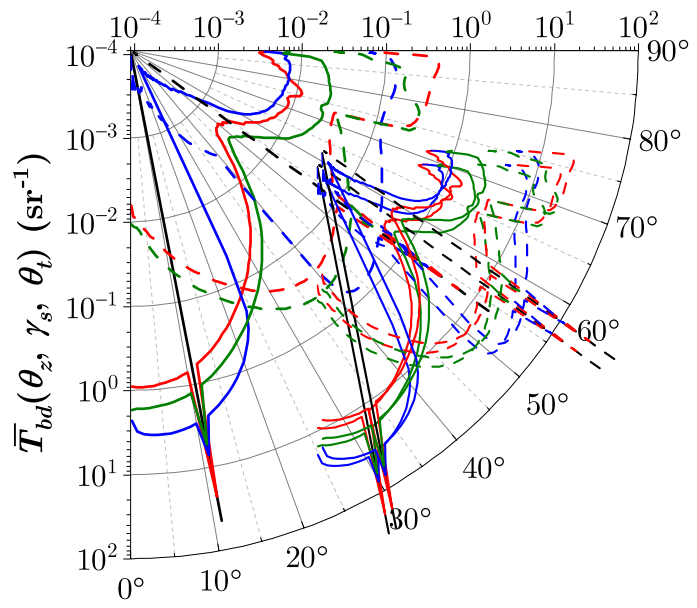

(c) June $21^{\text {st }}$

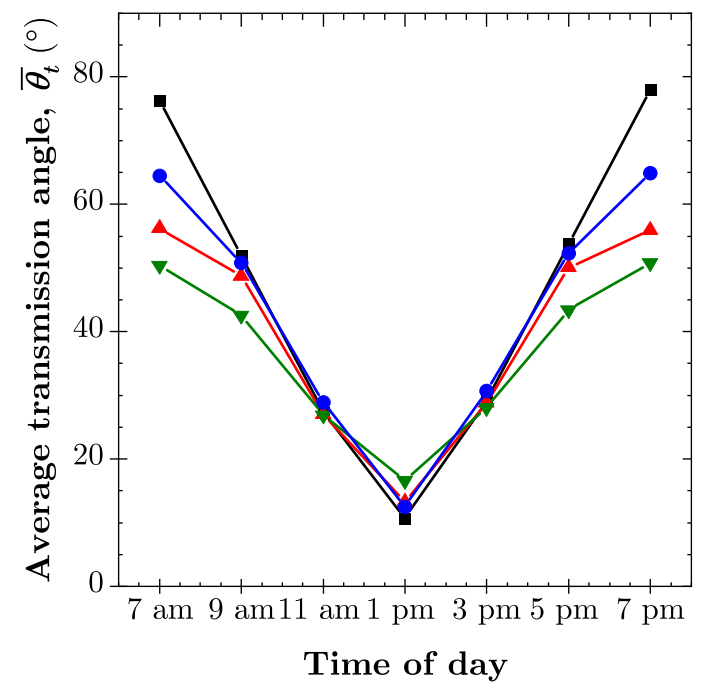

(e) June $21^{\text {st }}$

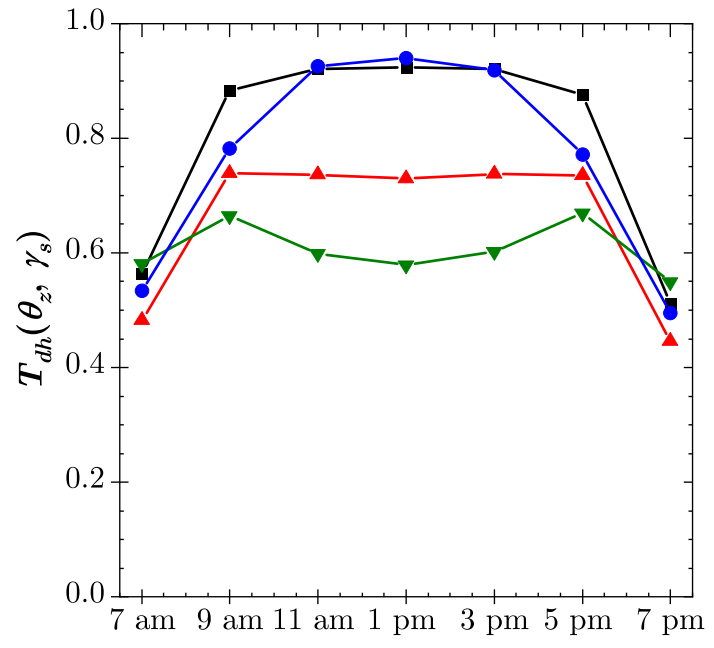

Time of day

\section{(b) September $23^{\text {rd }}$}
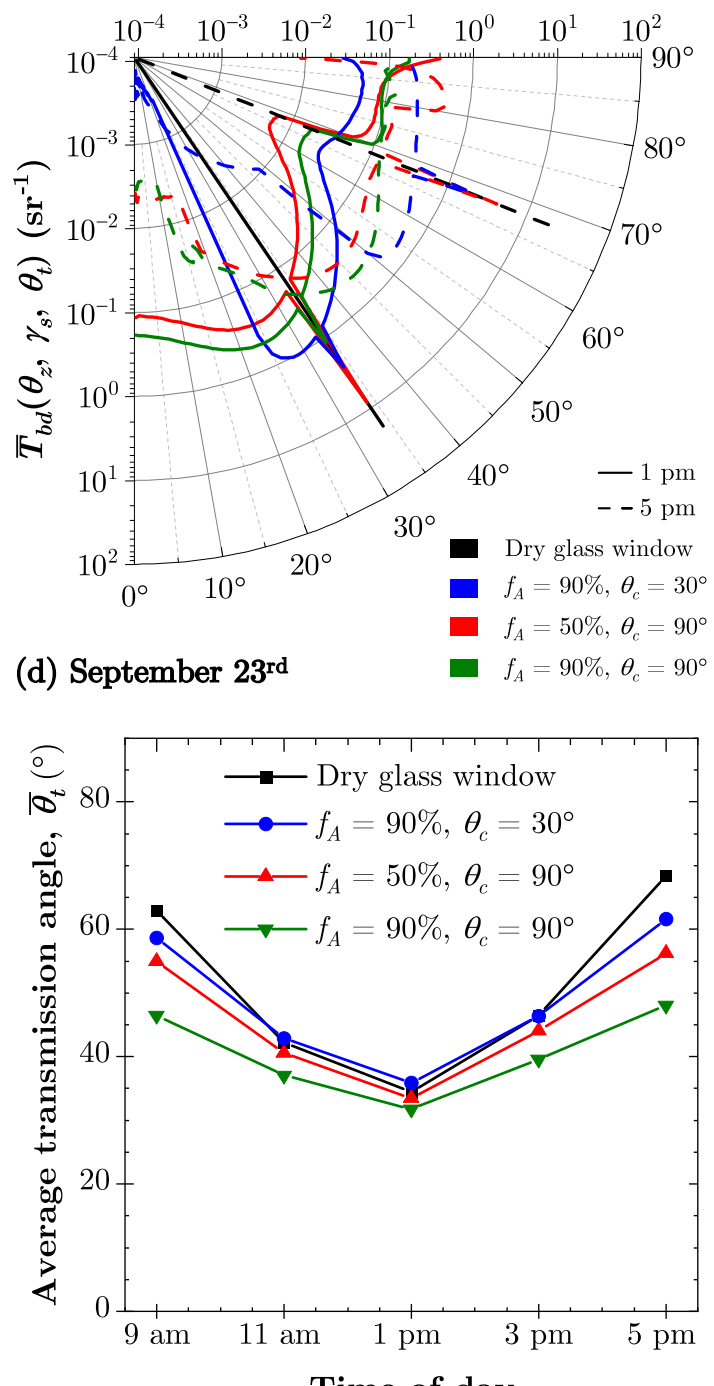

Time of day

(f) September $23^{\text {rd }}$

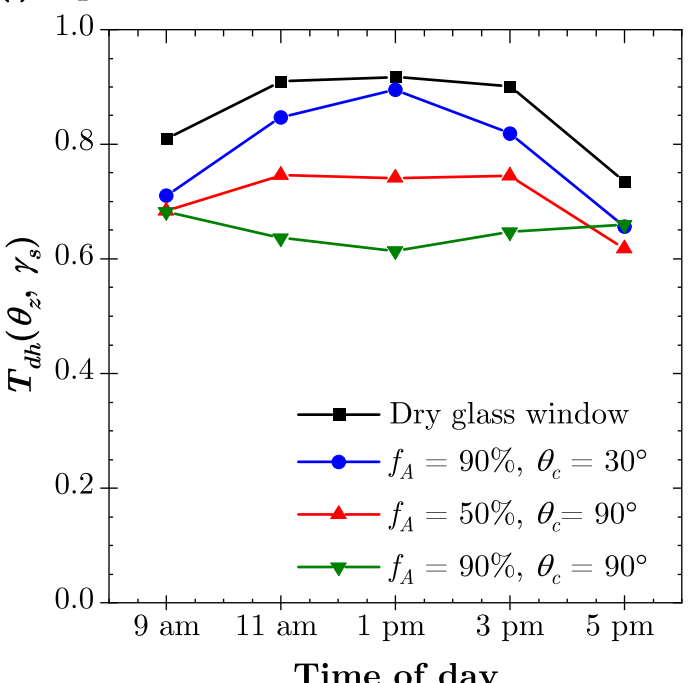

Figure 5. One-dimensional bidirectional transmittance $\bar{T}_{b d}$ at 1 p.m. and 5 p.m. (a,b), average transmission angle $\bar{\theta}_{t}(\mathbf{c}, \mathbf{d})$, and directional-hemispherical transmittance $T_{d h}\left(\theta_{z}, \gamma_{s}\right)(\mathbf{e}, \mathbf{f})$ as functions of time for a variety of window conditions on $(\mathbf{a}, \mathbf{c}, \mathbf{e}) 21$ June and $(\mathbf{b}, \mathbf{d}, \mathbf{f}) 23$ September. 
Finally, Figure 5e,f show the temporal evolution of the directional-hemispherical transmittance $T_{d h}$ for various window conditions on 21 June and 23 September, respectively. On both days, Figure $5 \mathrm{e}, \mathrm{f}$ indicate that the directional-hemispherical transmittance $T_{d h}$ for the dry window increased to reach a maximum around midday, before decreasing in the afternoon. This was due to the larger reflectance of glass at larger incidence angles [33]. Furthermore, Figure $5 \mathrm{e}, \mathrm{f}$ show that the presence of droplets reduced $T_{d h}$ for nearly all solar positions investigated on both days. Indeed, droplets with contact angle $\theta_{c}=90^{\circ}$ and surface area coverage $f_{A}=90 \%$ reduced $T_{d h}$ by up to $37 \%$ on 21 June. However, on the same day, $T_{d h}$ increased slightly compared with a dry window for droplet contact angle $\theta_{c}=30^{\circ}$ and surface area coverage $f_{A}=90 \%$ at 11 a.m. and 1 p.m. and for $\theta_{c}=90^{\circ}$ and $f_{A}=90 \%$ at 7 a.m. and 7 p.m. This was due to the smaller refractive index mismatch between the window $\left(n_{w}=1.5\right)$ and the water droplets $\left(n_{d}=1.33\right)$ for droplet-covered windows than between the window $\left(n_{w}=1.5\right)$ and the air $\left(n_{a}=1.0\right)$ for dry windows. Then, the presence of droplets reduced reflection at the back side of the window, resulting in slightly larger $T_{d h}$ compared to the dry window case. In addition, Figure 5 indicates that for droplet contact angle $\theta_{c}=90^{\circ}$, increasing the droplet surface area coverage $f_{A}$ resulted in smaller transmittance $T_{d h}$ of the PBR window between 9 a.m. and 5 p.m. on 21 June and at all times except 5 p.m. on 23 September. Moreover, under normal incidence, previous studies have shown that for a given surface area coverage $f_{A}$, the normal-hemispherical transmittance $T_{n h}$ decreased significantly with increasing droplet contact angle $\theta_{c}$ greater than the critical angle $\theta_{c r}$ for total internal reflection at the droplet/air interface such that $\theta_{c r}=\sin ^{-1}\left(1 / n_{d}\right)=48.8^{\circ}$ [8]. This phenomenon was also observed in Figure $5 \mathrm{e}, \mathrm{f}$ at near-normal incidence angles (i.e., small solar zenith angles $\theta_{z}$ ).

\subsection{Light Transfer in Microalgae Culture}

Figure $6 \mathrm{a}, \mathrm{b}$ show the normalized LRPA $\mathcal{A} / \mathcal{A}_{\mathcal{c}}$ as a function of the normalized culture depth $z / L$ for various window conditions at 1 p.m. and 5 p.m. in Los Angeles, CA, on 21 June and 23 September, respectively. Here, the initial biomass concentration of the PBR was $X_{0}=0.07 \mathrm{~g} \mathrm{~L}^{-1}$, and the culture depth was $L=0.3 \mathrm{~m}$. For both days, Figure 6 indicates that the normalized LRPA $\mathcal{A} / \mathcal{A}_{c}$ exceeded 1.0 at 1 p.m. throughout the culture while at 5 p.m. up to $58 \%$ of the culture volume features $\mathcal{A} / \mathcal{A}_{c}<1.0$, i.e., an illuminated fraction $\gamma$ as low as 0.42 . This was caused by (i) the smaller solar irradiance later in the day, (ii) the increase in the pathlength of light through the culture for larger solar zenith angles $\theta_{z}$, and (iii) the photosynthetic response that occurred between 1 p.m. and 5 p.m. Thus, more of the incident radiation was absorbed at shallower culture depths at 5 p.m. than at 1 p.m. These observations were also reported in previous studies $[9,25]$. On both days at 1 p.m., the droplets were responsible for a decrease in the local rate of photon absorption throughout the PBR depth, with the exception of a window with $f_{A}=90 \%$ and $\theta_{c}=30^{\circ}$ on 21 June at 1 p.m. due to the increase in $T_{d h}$ observed in Figure 5e. On the other hand, on both days at 5 p.m., Figure 6 shows that the presence of droplets with contact angle $\theta_{c}=90^{\circ}$ resulted in an increase of up to a $14 \%$ in the illuminated fraction $\gamma$ of the PBR, compared to a dry window. This was due to light scattering by the droplets observed in Figure $5 \mathrm{a}-\mathrm{d}$, which redistributed the incoming light in many directions and caused light to penetrate deeper into the culture. 
(a) June $21^{\text {st }}$

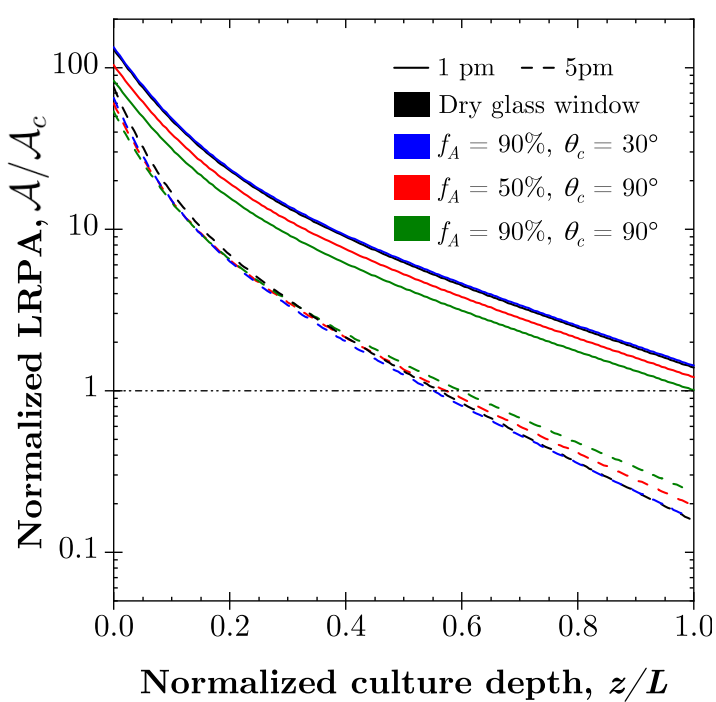

(b) September $23^{\text {rd }}$

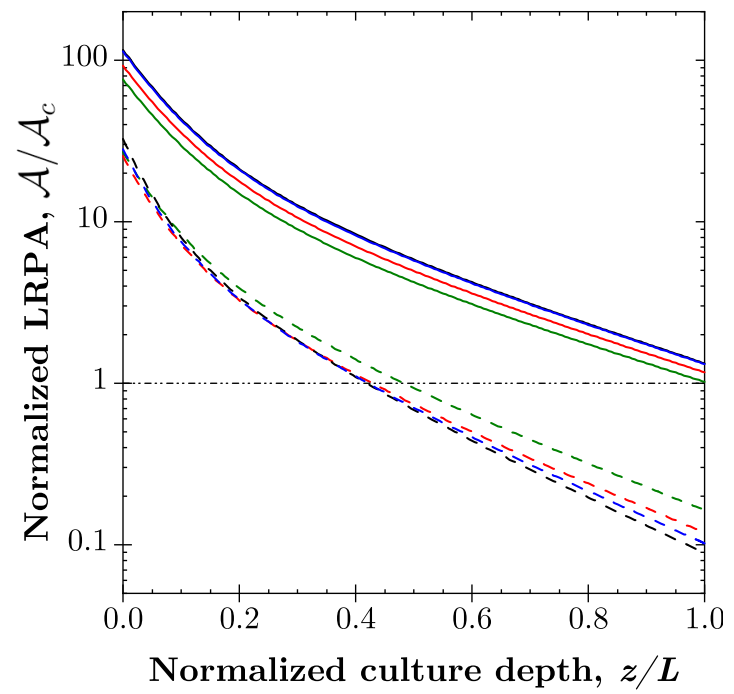

Figure 6. Normalized local rate of photon absorption (LRPA) $\mathcal{A} / \mathcal{A}_{c}$ as a function of the normalized culture depth $z / L$ at 1 p.m. and 5 p.m. for a variety of window conditions on (a) 21 June and (b) 23 September in Los Angeles, CA. Here, $L=0.3$ and $\mathcal{A}_{c}=2800 \mu \mathrm{mol}_{h v} \mathrm{~kg}^{-1} \mathrm{~s}^{-1}$.

\subsection{Microalgae Growth Kinetics}

Figure $7 \mathrm{a}, \mathrm{b}$ plot the temporal evolution of the average growth rate $\bar{\mu}(t)\left(\mathrm{in}^{-1}\right)$ for PBRs with various window conditions on 21 June and 23 September, respectively. Here also, the initial biomass concentration $X_{0}$ was $0.07 \mathrm{~g} \mathrm{~L}^{-1}$, and the culture depth $L$ was $0.3 \mathrm{~m}$. For most of the day on 21 June, Figure $7 \mathrm{a}$ indicates that droplets reduced the average growth rate $\bar{\mu}(t)$. Furthermore, the average growth rate $\bar{\mu}(t)$ decreased with increasing droplet contact angle $\theta_{\mathcal{C}}$ and surface area coverage $f_{A}$. A similar trend was observed on 23 September. However, at 5 p.m. on 23 September and 7 a.m. and 7 p.m. on 21 June, droplets with $\theta_{\mathcal{c}}=90^{\circ}$ and $f_{A}=90 \%$ slightly increased $\bar{\mu}(t)$. This was attributed to the positive effect of light scattering by droplets towards the culture observed previously, which increased the illuminated fraction $\gamma$ and light penetration in the culture late in the day (see Figure 6). Interestingly, on 23 September, this was the case despite a relative decrease in the directional-hemispherical transmittance $T_{d h}$ of up to $24 \%$ compared to a dry window. Nonetheless, this phenomena occurred in the morning and evening, when the average growth rate $\bar{\mu}(t)$ was a fraction of its daily peak value and the impact on the final biomass concentration $X_{f}$ was minimal.

Indeed, Figure $7 \mathrm{c}, \mathrm{d}$ plot the temporal evolution of the biomass concentration $X(t)$ as a function of time for various window conditions on 21 June and 23 September, respectively. The figures indicate that the final biomass concentration $X_{f}$ was smaller on 23 September compared to 21 June for all configurations considered. This was due to the decrease in spectral solar irradiance $G_{S, \lambda}$ on 23 September (see Figure $3 a, b$ ) and the larger solar zenith angles $\theta_{z}$ (see Table 1), which led to a smaller window transmittance $T_{d h}$ in the presence of droplets. The presence of droplets decreased the final biomass concentration $X_{f}$ by up to $10.0 \%$ and $8.2 \%$ on 21 June and 23 September, respectively. 
(a) June $21^{\text {st }}$

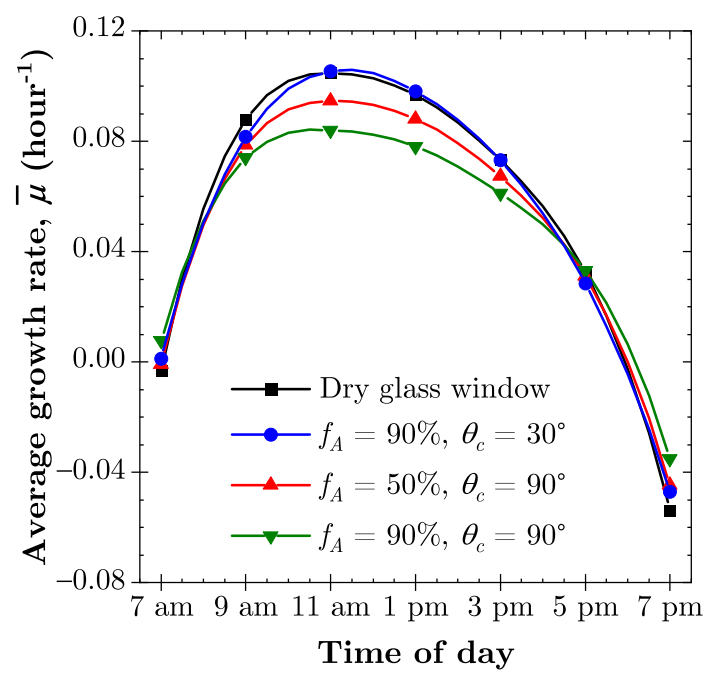

(c) June $21^{\text {st }}$

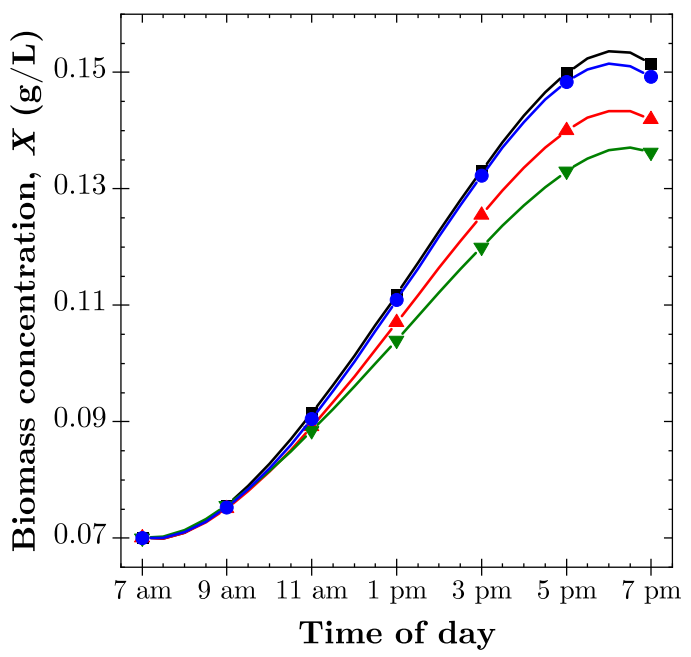

(b) September $2^{\text {rd }}$

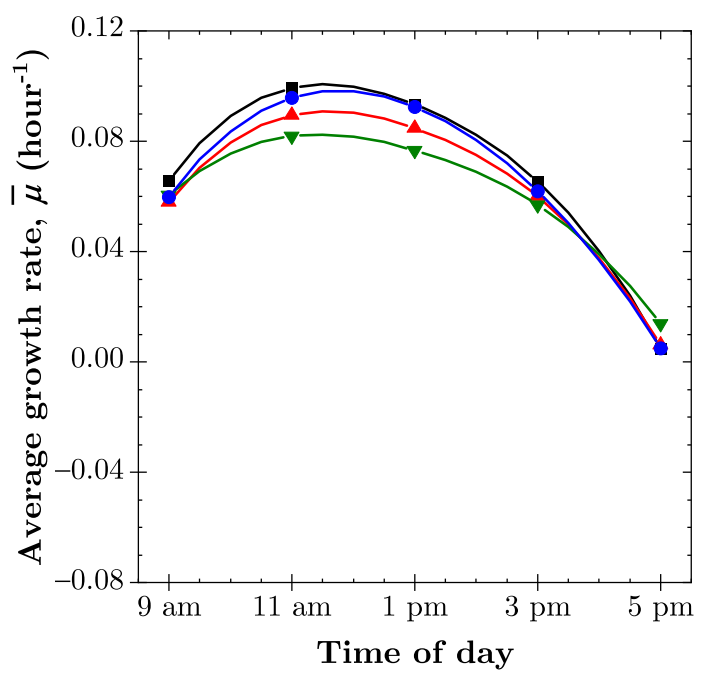

(d) September $\mathbf{2 3}^{\text {rd }}$

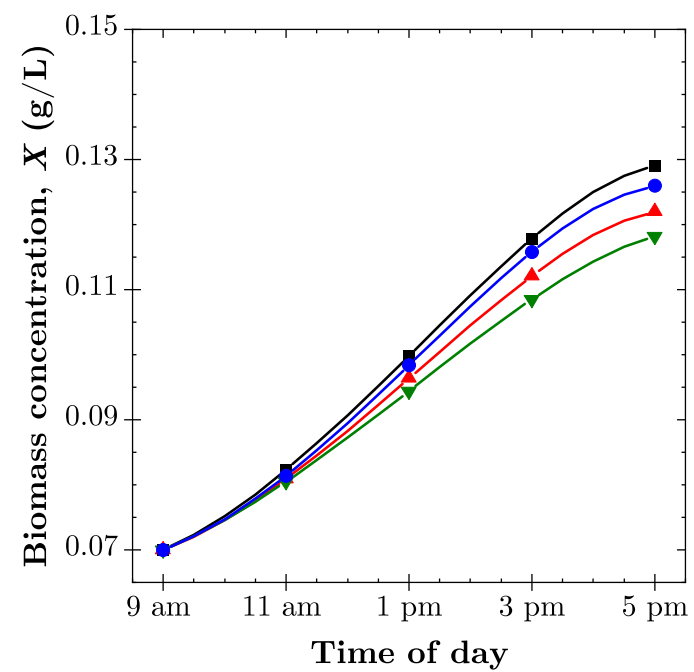

Figure 7. Average PBR growth rate $\bar{\mu}$ as a function of time for various window conditions on (a) 21 June and (b) 23 September and corresponding biomass concentration $X(t)$ on (c) 21 June and (d) 23 September.

\subsection{PBR Biomass Productivity}

Figure $8 \mathrm{a}, \mathrm{b}$ show the daily areal biomass productivity $P\left(\right.$ in $\mathrm{kg} \mathrm{m}^{-2}$ day $^{-1}$ ) as a function of the initial biomass concentration $X_{0}$ for various culture depths $L$ and window conditions for PBR operation between 7 a.m. and 7 p.m. on 21 June and 9 a.m. and 5 p.m. on 23 September, respectively. The trends in the daily biomass productivity $P$ were similar for both days although $P$ was smaller on 23 September than on 21 June. On both days, for a given culture depth $L$, the culture did not fully absorb the incoming radiation for small initial biomass concentrations $X_{0}$. However, as $X_{0}$ increased, more of the incoming light was absorbed by the culture, and $P$ increased to reach a maximum $P_{\max }$. As previously discussed, the optimal biomass productivity occurs for a light-limited PBR when the incoming radiation is fully absorbed in the culture, i.e., the microalgae suspension is fully illuminated with no dark zones. As $X_{0}$ increased further, dark zones appeared in the culture causing the areal biomass productivity $P$ to decrease. Figure $8 \mathrm{a}, \mathrm{b}$ indicate that as the culture depth $L$ increased, the maximum biomass productivity occurred for smaller values of $X_{0}$. 
Lee et al. [22] demonstrated that PBR productivity $P$ scaled with $X_{0} / a$ where $a$ is the specific illuminated area. In the case of the simulated raceway pond, $a$ is given by $a=S / V=1 / L$ where $S$ is the illuminated surface area and $V$ is the culture volume [30]. Furthermore, we note that the initial optical thickness of the PBR is defined as $\beta_{\lambda, 0} L=\left(\bar{A}_{a b s, \lambda}+\bar{S}_{s c a, \lambda}\right) X_{0} L$ where $\beta_{\lambda, 0}$ is the initial culture extinction coefficient (in $\left.\mathrm{m}^{-1}\right)$. Thus, the quantity $X_{0} L=X_{0} / a$ is representative of the culture initial optical thickness [22]. In order to test this scaling relationship for PBRs covered by windows supporting pendant droplets, Figure $8 \mathrm{c}$,d plots the biomass productivity $P$ with respect to the initial optical thickness represented by $X_{0} / a$ for various window conditions. Both figures indicate that, for a given day and window condition, all data collapse on the same line. Thus, the biomass productivity $P$ depended solely on $X_{0} / a$, the PBR window conditions, and the day of the year. Furthermore, for a given day of the year, the optimum initial optical thickness $\left(X_{0} / a\right)_{\text {opt }}$ corresponding to the maximum biomass productivity $P_{\text {max }}$ was essentially independent of the window condition. Note that the same results were obtained by Lee et al. [22], albeit using a different growth kinetics model for open ponds, as well as vertical and tubular PBRs without droplets cultivating Chlamydomonas reinhardtii. These results confirm the importance of the optical thickness represented by $X_{0} / a$ for maximizing PBR biomass productivity through the PBR design (via $a=1 / L$ ) and its operation (via $X_{0}$ ) regardless of the window condition and/or growth kinetics model. Figure $8 \mathrm{~d}$ shows that on 23 September, all droplet configurations decreased the maximum biomass productivity $P_{\text {max }}$. By contrast, Figure 8c shows that, on 21 June, the effect of droplets with $\theta_{c}=30^{\circ}$ and $f_{A}=90 \%$ was essentially negligible, while droplets with $\theta_{c}=90^{\circ}$ decreased $P_{\text {max }}$.

Table 2 summarizes the maximum biomass productivity $P_{\max }$ and the corresponding optimum initial optical thickness $\left(X_{0} / a\right)_{\text {opt }}$ for each droplet configuration for both 21 June and 23 September, as well as the change in $P_{\max }$ relative to the reference case of a PBR covered by a dry window. It is evident that the droplet contact angle strongly affected the maximum achievable PBR biomass productivity $P_{\max }$. Indeed, the maximum biomass productivity $P_{\max }$ decreased by just $2.0 \%$ and $5.1 \%$ for droplet contact angle $\theta_{c}=30^{\circ}$ and surface area coverage $f_{A}=90 \%$ on 21 June and 23 September, respectively. On the other hand, $P_{\max }$ decreased by $17.5 \%$ and $18.1 \%$ for $\theta_{c}=90^{\circ}$ and $f_{A}=90 \%$ on 21 June and 23 September, respectively. Although hydrophobic surfaces may inhibit condensation [37], these results suggest that a hydrophilic cover featuring small droplet contact angle $\theta_{c}$ should be selected whenever possible to mitigate the effect of droplets on the light reaching the culture and on the PBR biomass productivity. Indeed, on a given day, the daily biomass productivity $P$ was larger for droplet contact angle $\theta_{c}=30^{\circ}$ and surface area coverage $f_{A}=90 \%$ than for $\theta_{c}=90^{\circ}$ and $f_{A}=50 \%$ on both days and for all values of optical thickness $X_{0} / a$. 
(a) June $21^{\text {st }}$

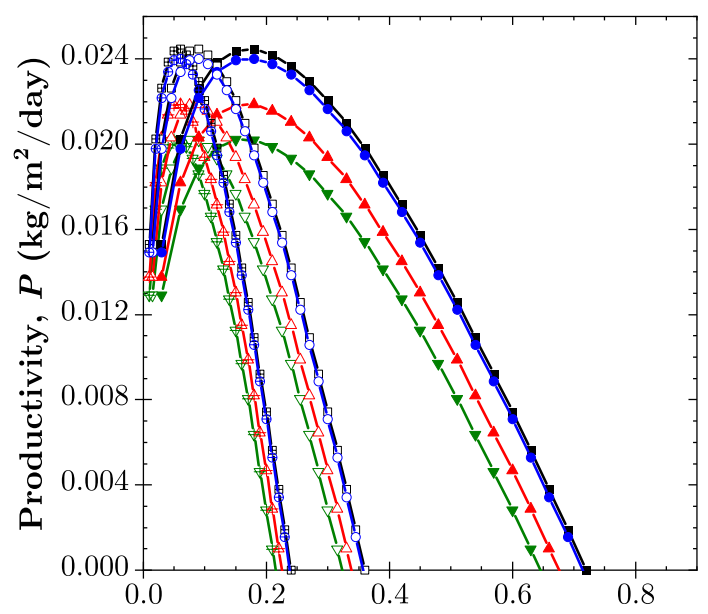

Initial biomass concentration, $X_{0}(\mathrm{~g} / \mathrm{L})$

(c) June $21^{\text {st }}$

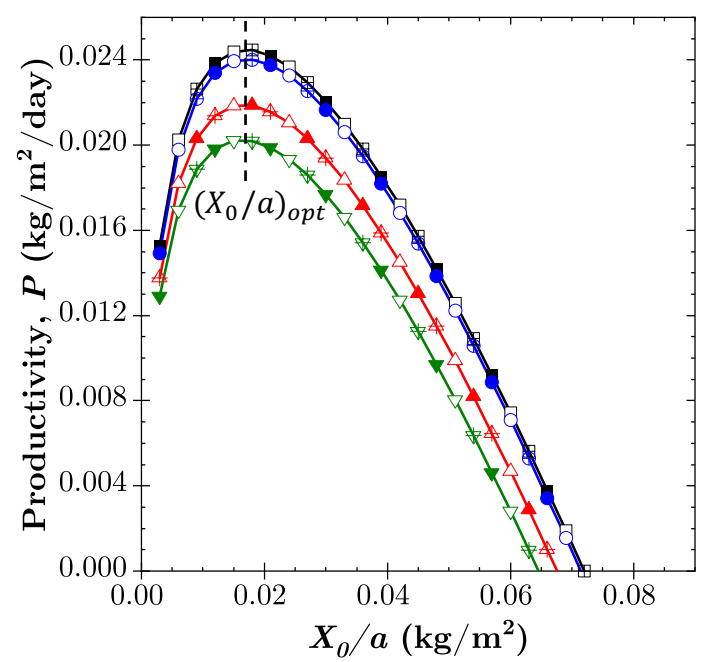

(b) September $\mathbf{2 3}^{\text {rd }}$

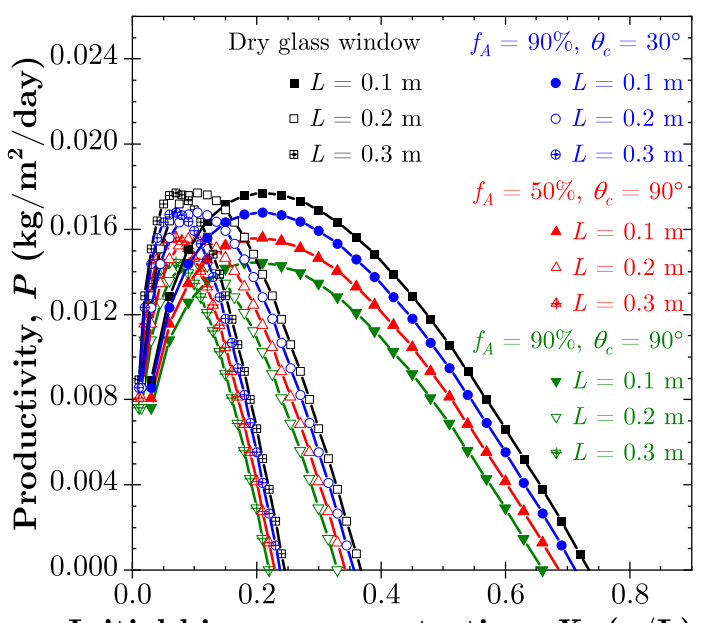

Initial biomass conentration, $\boldsymbol{X}_{0}(\mathrm{~g} / \mathrm{L})$

(d) September $23^{\text {rd }}$

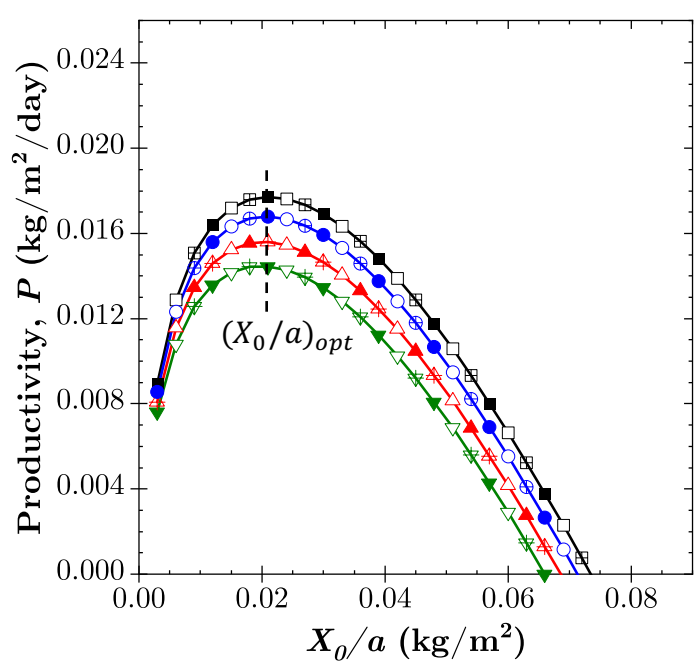

Figure 8. Daily areal PBR biomass productivity $P$ as a function of $(\mathbf{a}, \mathbf{b})$ starting biomass concentration $X_{0}$ for various PBR depths $L$ and window conditions and $(\mathbf{c}, \mathbf{d})$ the same daily areal PBR biomass productivity $P$ as a function of the microalgae culture initial optical thickness represented by $X_{0} / a$, on $(\mathbf{a}, \mathbf{c}) 21$ June and $(\mathbf{b}, \mathbf{d}) 23$ September 
Table 2. Maximum biomass productivity $P_{\max }$ and the corresponding optimum optical thickness $\left(X_{0} / a\right)_{\text {opt }}$ for each window condition on 21 June and 23 September. The percent change in $P_{\max }$ compared to that of a dry window $\left(f_{A}=0 \%\right)$ is also shown.

\begin{tabular}{|c|c|c|c|c|}
\hline \multicolumn{5}{|c|}{21 June } \\
\hline$f_{A}$ & $\theta_{c}$ & $\begin{array}{l}\left(X_{0} / a\right)_{o p t} \\
\left(\mathrm{~kg} \mathrm{~m}^{-2}\right)\end{array}$ & $\begin{array}{c}P_{\max } \\
\left(\mathrm{kg} \mathrm{m}^{-2} \mathrm{day}^{-1}\right)\end{array}$ & $\begin{array}{c}P_{\max } \\
\text { Change }\end{array}$ \\
\hline $0 \%$ & $\mathrm{~N} / \mathrm{A}$ & 0.018 & 0.0245 & $0 \%$ \\
\hline $90 \%$ & $30^{\circ}$ & 0.018 & 0.0240 & $-2.0 \%$ \\
\hline $50 \%$ & $90^{\circ}$ & 0.018 & 0.0219 & $-10.6 \%$ \\
\hline $90 \%$ & $90^{\circ}$ & 0.018 & 0.0202 & $-17.5 \%$ \\
\hline \multicolumn{5}{|c|}{23 September } \\
\hline$f_{A}$ & $\theta_{c}$ & $\begin{array}{l}\left(X_{0} / a\right)_{o p t} \\
\left(\mathrm{~kg} \mathrm{~m}^{-2}\right)\end{array}$ & $\begin{array}{c}P_{\max } \\
\left(\mathrm{kg} \mathrm{m}^{-2} \mathrm{day}^{-1}\right)\end{array}$ & $\begin{array}{c}P_{\max } \\
\text { Change }\end{array}$ \\
\hline $0 \%$ & $\mathrm{~N} / \mathrm{A}$ & 0.021 & 0.0177 & $0 \%$ \\
\hline $90 \%$ & $30^{\circ}$ & 0.021 & 0.0168 & $-5.1 \%$ \\
\hline $50 \%$ & $90^{\circ}$ & 0.021 & 0.0156 & $-11.9 \%$ \\
\hline $90 \%$ & $90^{\circ}$ & 0.021 & 0.0145 & $-18.1 \%$ \\
\hline
\end{tabular}

\section{Conclusions}

This study demonstrates the effect of condensate droplets on the biomass productivity of covered outdoor raceway ponds. Numerical simulations of light transfer were coupled to a microalgae growth kinetics to predict the performance of PBRs with and without condensate droplet-covered windows. The bidirectional transmittance of dropletcovered windows in Los Angeles, CA, at different times throughout the day on 21 June and 23 September was predicted by the Monte Carlo ray-tracing method. The local fluence rate within the horizontal culture of Chlorella vulgaris was predicted using the two-flux approximation for oblique incidence. A growth kinetics model accounting for photolimitation, photoinhibition, and respiration was applied. Droplets with contact angle $\theta_{c}=90^{\circ}$ decreased the maximum areal PBR biomass productivity $P_{\max }$ by up to $17.5 \%$ and $18.1 \%$ on 21 June and 23 September, respectively. On both days, the maximum daily areal PBR biomass productivity decreased with increasing droplet contact angle and surface area coverage compared with a dry window. Thus, hydrophilic PBR covers are preferable to minimize the adverse impacts of droplets on PBR biomass productivity. Additionally, the areal PBR biomass productivity scaled with the ratio $X_{0} / a$ of the initial biomass concentration $X_{0}$ and the specific illuminated area $a=1 / L$ such that $X_{0} / a=X_{0} L$ was proportional to the initial optical thickness of the PBR. Finally, for a given day of the year, the maximum biomass productivity occurred at the same initial optical thickness for all window conditions simulated. This optimal initial optical thickness can be used to maximize PBR biomass productivity through PBR design (via $L$ ) and operation (via $X_{0}$ ).

Author Contributions: Conceptualization, J.P., J.L., E.-k.S.-A., and L.P.; data curation, J.H., K.Z. and J.P.; formal analysis, J.H. and K.Z.; funding acquisition, L.P.; investigation, J.H. and K.Z.; methodology, J.H., K.Z., J.P., and L.P.; software, J.H., K.Z., and J.P.; supervision, J.P., J.L., and L.P.; validation, J.H. and K.Z.; visualization, J.H. and L.P.; writing, original draft, J.H.; writing, review and editing, J.H., J.P., J.L., E.-k.S.-A., and L.P. All authors read and agreed to the published version of the manuscript.

Funding: This research was supported in part by the National Science Foundation NRT-INFEWS: Integrated Urban Solutions for Food, Energy, and Water Management (Grant No. DGE-1735325) and the DISCUS project of the NExT Initiative (Nantes Excellence Trajectory) International Research Partnership.

Institutional Review Board Statement: Not applicable.

Informed Consent Statement: Not applicable. 
Data Availability Statement: Data presented in this manuscript are available upon request from the corresponding author.

Conflicts of Interest: The authors declare no conflict of interest. The funders had no role in the design of the study; in the collection, analyses, or interpretation of data; in the writing of the manuscript; nor in the decision to publish the results.

\section{Nomenclature}

$\bar{A}_{a b s, \lambda}$

$\mathcal{A}(z)$

$\mathcal{A}_{c}$

$b_{\lambda}$

$d$

$d_{p}$

$f_{A}$

G

$H$

I

$\mathrm{JNADH}_{2}$

$\mathrm{JO}_{2}(z)$

$\bar{J}_{\mathrm{O}_{2}}$

$k$

K

$K_{r}$

$L$

M

$M_{x}$

$N$

$n$

$P$

$q$

$S$

$\bar{S}_{s c a, \lambda}$

$t$

$T_{b d}$

$\bar{T}_{b d}$

$T_{d h}$

$T_{n h}$

V

$X$

$z$ specific illuminated area, $\mathrm{m}^{-1}$

average spectral mass absorption cross-section, $\mathrm{m}^{2} \mathrm{~kg}^{-1}$

local specific rate of photon absorption, $\mu \mathrm{mol}_{h v} \mathrm{~kg}^{-1} \mathrm{~s}^{-1}$

specific rate of photon absorption at the compensation point, $\mu \mathrm{mol}_{h v} \mathrm{~kg}^{-1} \mathrm{~s}^{-1}$

spectral back-scattering ratio

droplet diameter, $\mu \mathrm{m}$

droplet projected diameter, $\mu \mathrm{m}$

droplet surface area coverage, $\%$

fluence rate, $\mu_{\mathrm{mol}} \mathrm{m}^{-2} \mathrm{~s}^{-1}$

window thickness, $\mathrm{m}$

radiative intensity, $\mu \mathrm{mol}_{h v} \mathrm{~m}^{-2} \mathrm{~s}^{-1} \mathrm{sr}^{-1}$

specific rate of cofactor regeneration, $\operatorname{mol}_{\mathrm{NADH}_{2}} \mathrm{~kg}_{\mathrm{X}}^{-1} \mathrm{~s}^{-1}$

local specific rate of oxygen production/consumption,

in mol $_{\mathrm{O}_{2}} \mathrm{~kg}_{\mathrm{X}}^{-1} \mathrm{~s}^{-1}$

average specific rate of oxygen production/consumption, $\operatorname{mol}_{\mathrm{O}_{2}} \mathrm{~kg}_{\mathrm{x}}^{-1} \mathrm{~s}^{-1}$

absorption index

half saturation constant for photosynthesis, $\mu \mathrm{mol}_{h v} \mathrm{~kg}^{-1} \mathrm{~s}^{-1}$

half saturation constant for respiration, $\mu_{\mathrm{mol}} \mathrm{kg}^{-1} \mathrm{~s}^{-1}$

microalgae culture depth, $\mathrm{m}$

interval number of transmission angles

carbon molar mass in the biomass, $\mathrm{kg}_{\mathrm{x}} \mathrm{mol}_{\mathrm{C}}^{-1}$

number of rays

refractive index

daily PBR biomass productivity, $\mathrm{kg} \mathrm{m}^{-2}$ day $^{-1}$

radiative flux, $\mu \mathrm{mol}_{h v} \mathrm{~m}^{-2} \mathrm{~s}^{-1}$

culture illuminated surface area, $\mathrm{m}^{2}$

average spectral mass scattering cross-section, $\mathrm{m}^{2} \mathrm{~kg}^{-1}$

time, hour

bidirectional transmittance, $\mathrm{sr}^{-1}$

one-dimensional bidirectional transmittance, $\mathrm{sr}^{-1}$

directional-hemispherical transmittance

normal-hemispherical transmittance

culture volume, $\mathrm{m}^{3}$

biomass concentration, $\mathrm{g} \mathrm{L}^{-1}$

culture depth, $\mathrm{m}$ 


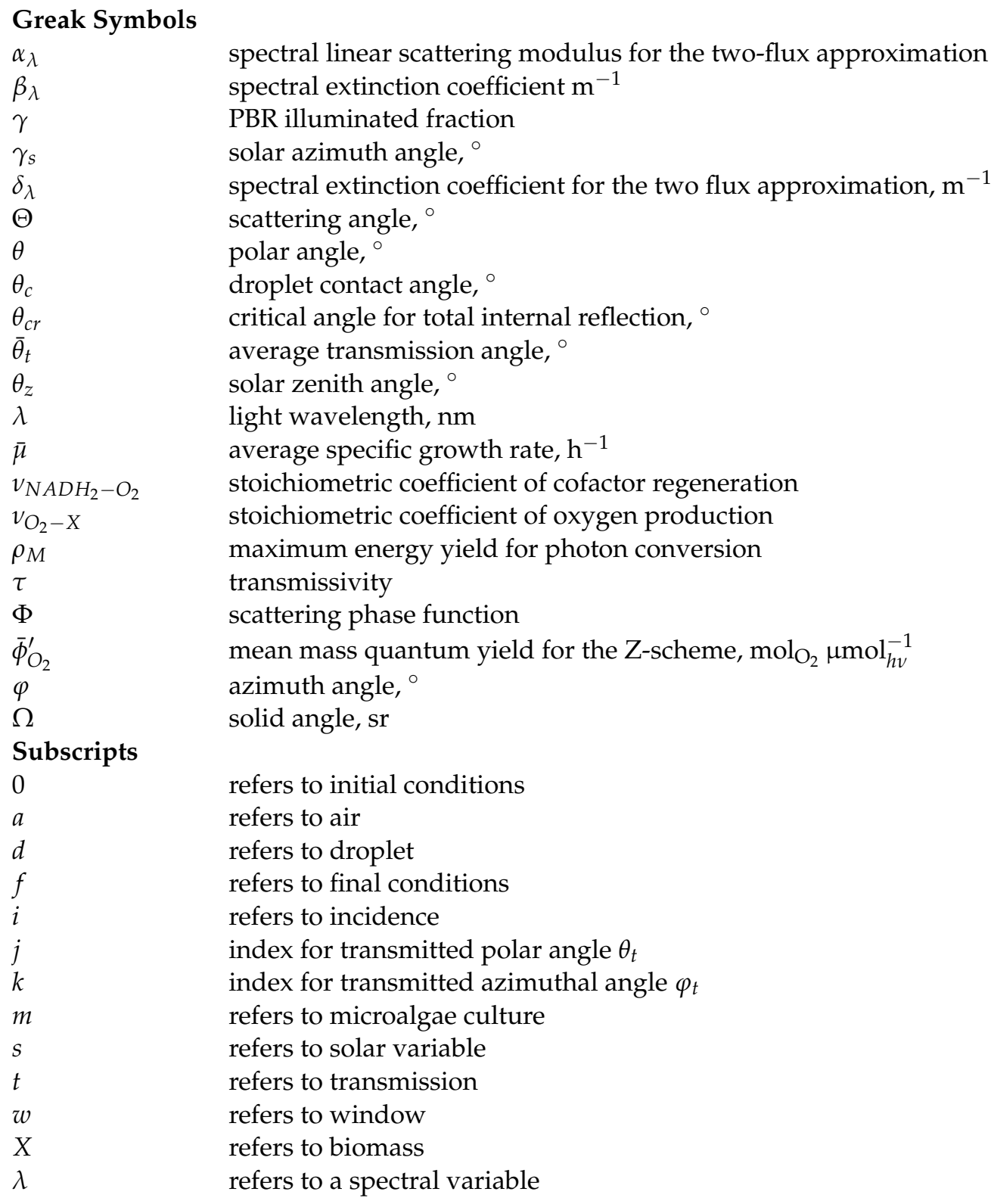

\section{Appendix A}

Table A1. Kinetic growth parameters of Chlorella vulgaris from Ref. [9].

\begin{tabular}{ccc}
\hline Parameter & Value & Units \\
\hline$\rho_{M}$ & 0.8 & - \\
$J_{N A D H_{2}}$ & $2.8 \times 10^{-3}$ & $\operatorname{mol}_{\mathrm{NADH}_{2}} \mathrm{~kg}_{\mathrm{X}}^{-1} \mathrm{~s}^{-1}$ \\
$v_{\mathrm{O}_{2}-X}$ & 1.13 & - \\
$\bar{\phi}^{\prime} \mathrm{O}_{2}$ & $1.1 \times 10^{-7}$ & $\mathrm{~mol}_{\mathrm{O}_{2}} \mu \mathrm{mol}_{h v}^{-1}$ \\
$M_{X}$ & 0.024 & $\mathrm{~kg}_{\mathrm{x}} \mathrm{mol}_{\mathrm{C}}^{-1}$ \\
$v_{N A H_{2}-\mathrm{O}_{2}}$ & 2 & - \\
$K$ & 40,000 & $\mu \mathrm{mol}_{h v} \mathrm{~kg}^{-1} \mathrm{~s}^{-1}$ \\
$K_{r}$ & 556.5 & $\mu \mathrm{mol}_{h v} \mathrm{~kg}^{-1} \mathrm{~s}^{-1}$ \\
$\mathcal{A}_{c}$ & 2800 & $\mu \mathrm{mol}_{h v} \mathrm{~kg}^{-1} \mathrm{~s}^{-1}$
\end{tabular}


(a)

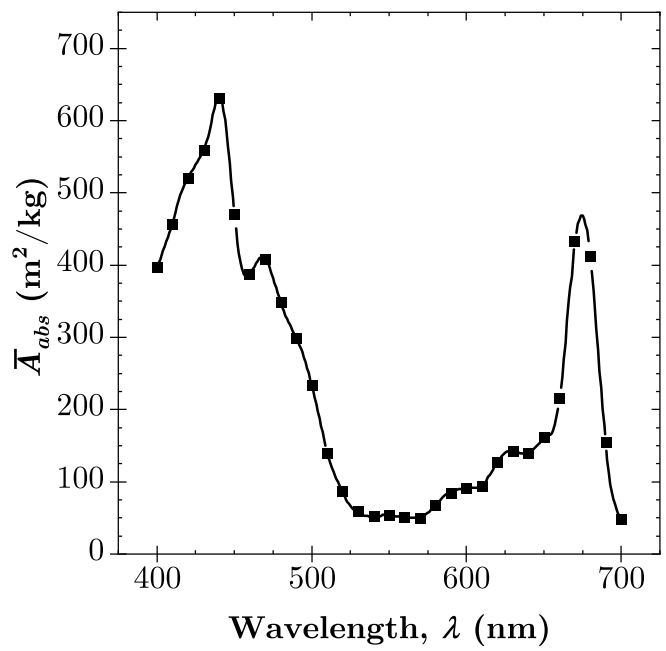

(b)

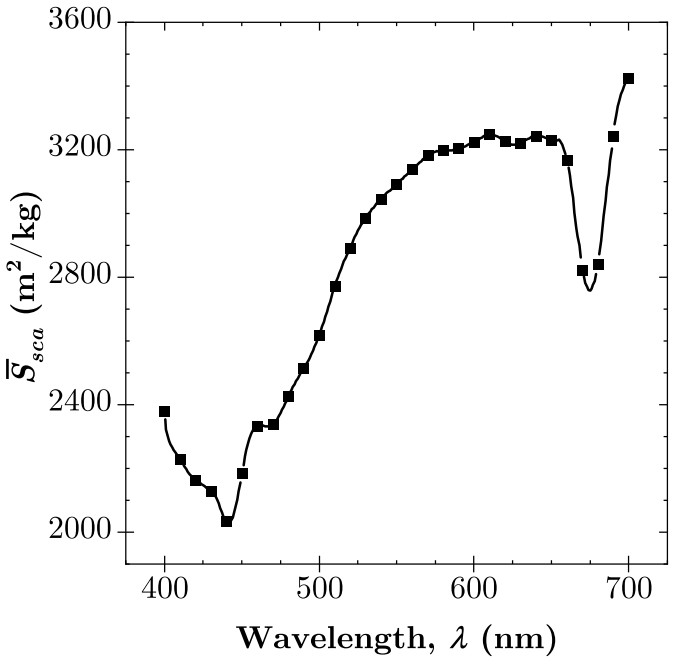

(c)

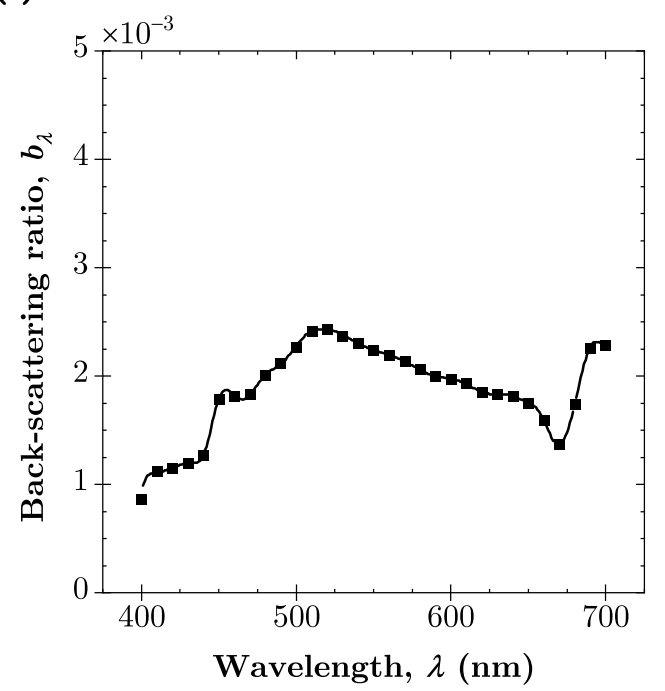

Figure A1. Average spectral mass (a) absorption $\bar{A}_{a b s, \lambda}$ and (b) scattering $\bar{S}_{s c a, \lambda}$ cross-sections taken from [9] for Chlorella vulgaris with a mean cell radius of $2 \mu \mathrm{m}$ and chlorophyll $a$, chlorophyll $b$, and photoprotective carotenoid (PPC) concentrations of $3.3 \mathrm{wt} . \%, 0.85 \mathrm{wt} . \%$, and $0.85 \mathrm{wt} . \%$, respectively. (c) Spectral backward scattering ratio $b_{\lambda}$ as a function of wavelength $\lambda$ calculated using the method presented in [35]. 
(a) June $21^{\text {st }}$

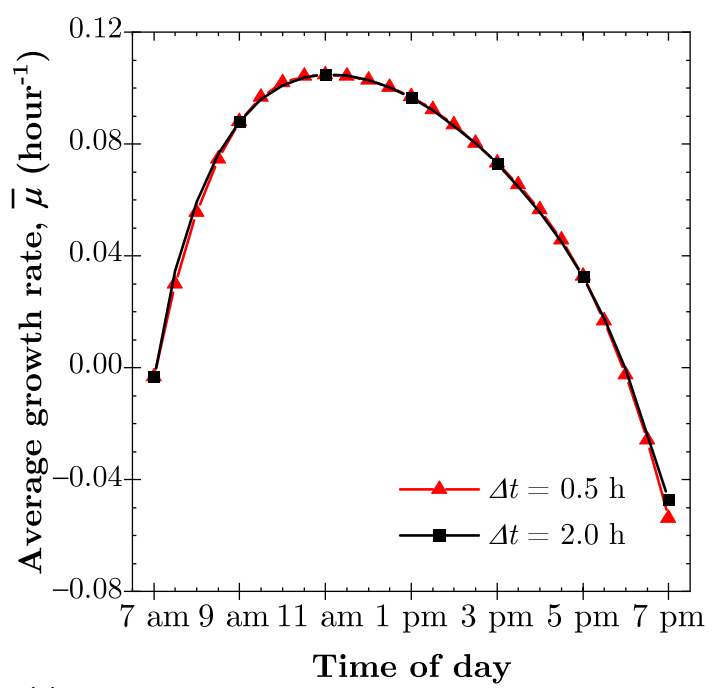

(c) June $21^{\text {st }}$

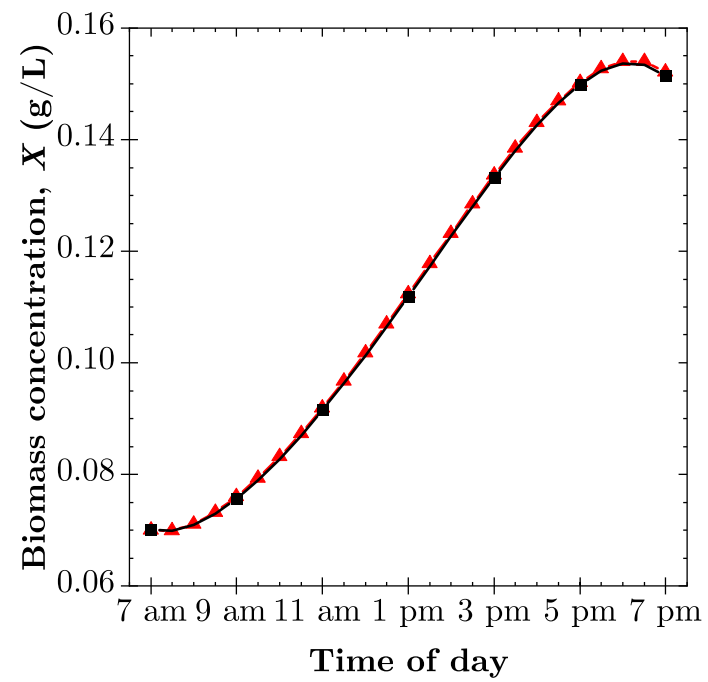

(b) September $23^{\text {rd }}$

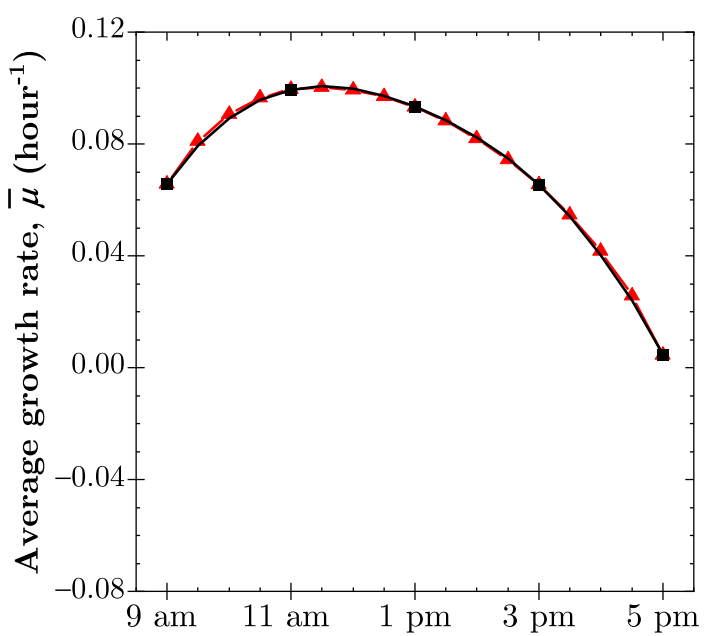

Time of day

(d) September $23^{\text {rd }}$

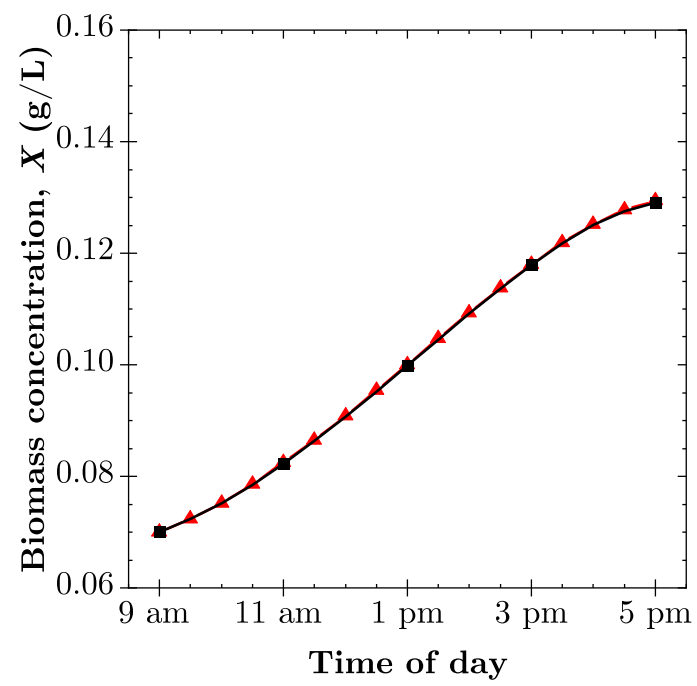

Figure A2. Comparison of the average specific growth rate $\bar{\mu}(t)$ on (a) 21 June and (b) 23 September and the biomass concentration $X(t)$ on (c) 21 June and (d) 23 September for droplet-free PBRs simulated using a time increment $\Delta t$ of $0.5 \mathrm{~h}$ and $2 \mathrm{~h}$. The PBRs had an initial biomass concentration $X_{0}$ of $0.07 \mathrm{gL}^{-1}$ and a culture depth $L$ of $0.3 \mathrm{~m}$. The maximum relative error in the biomass concentration $X(t)$ was $0.1 \%$ and $0.3 \%$ on 21 June and 23 September, respectively.

\section{References}

1. Chisti, Y. Biodiesel from microalgae. Biotechnol. Adv. 2007, 25, 294-306. [CrossRef] [PubMed]

2. Chew, K.W.; Yap, J.Y.; Show, P.L.; Suan, N.H.; Juan, J.C.; Ling, T.C.; Lee, D.J.; Chang, J.S. Microalgae biorefinery: High value products perspectives. Bioresour. Technol. 2017, 229, 53-62. [CrossRef] [PubMed]

3. Tredici, M.R. Mass Production of Microalgae: Photobioreactors. In Handbook of Microalgal Culture: Biotechnology and Applied Phycology; Richmond, A., Ed.; Blackwell Publishing Ltd.: Cornwall, UK, 2004; Chapter 9; pp. 178-214.

4. Pruvost, J.; Cornet, J.-F.; Pilon, L. Large-scale production of algal biomass: Photobioreactors. In Algae Biotechnology: Products and Processes; Bux, F., Chisti, Y., Eds.; Springer International Publishing: Cham, Switzerland, 2016; pp. 41-66.

5. Pruvost, J.; Borgne, F.; Artu, A.; Legrand, J. Development of a thin-film solar photobioreactor with high biomass volumetric productivity (AlgoFilm $($ ) based on process intensification principles. Algal Res. 2017, 21, 120-137. [CrossRef]

6. Grobbelaar, J.U. Factors governing algal growth in photobioreactors: The "open" versus "closed" debate. J. Appl. Phycol. 2009, 21, 489-492. [CrossRef]

7. Huang, Y.; Feng, C.; Hoeniges, J.; Zhu, K.; Pilon, L. Bidirectional transmittance of transparent windows with external or backside condensation of nonabsorbing cap-shaped droplets. J. Quant. Spectrosc. Radiat. Transf. 2020, 251, 107039. [CrossRef]

8. Zhu, K.; Pilon, L. Transmittance of semitransparent windows with non-absorbing cap-shaped droplets condensed on their backside. J. Quant. Spectrosc. Radiat. Transf. 2017, 194, 98-107. [CrossRef] 
9. Souliès, A.; Legrand, J.; Marec, H.; Pruvost, J.; Castelain, C.; Burghelea, T.; Cornet, J.F. Investigation and modeling of the effects of light spectrum and incident angle on the growth of Chlorella Vulgaris in photobioreactors. Biotechnol. Prog. 2016, 32, $247-261$. [CrossRef]

10. Hsieh, C.K.; Rajvanshi, A.K. The effect of dropwise condensation on glass solar properties. Sol. Energy 1997, 19, 389-393. [CrossRef]

11. Briscoe, B.J.; Galvin, K.P. The effect of surface fog on the transmittance of light. Sol. Energy 1991, 46, 191-197. [CrossRef]

12. Zhu, K.; Li, S.; Pilon, L. Light transfer through windows with external condensation. J. Quant. Spectrosc. Radiat. Transf. 2018, 208, 164-171. [CrossRef]

13. Pollet, I.V.; Pieters, J.G. Condensation and radiation transmittance of greenhouse cladding materials, Part 3: Results for glass plates and plastic films. J. Agric. Eng. Res. 2000, 77, 419-428. [CrossRef]

14. Pollet, I.V.; Pieters, J.G. Condensation and radiation transmittance of greenhouse cladding materials, Part 2: Results for a complete condensation cycle. J. Agric. Eng. Res. 2000, 75, 65-72. [CrossRef]

15. Pollet, I.V.; Pieters, J.G. PAR transmittances of dry and condensate covered glass and plastic greenhouse cladding. Agric. For. Meteorol. 2002, 110, 285-298. [CrossRef]

16. Bhardwaj, R.; Kortenaar, M.V.t.; Mudde, R.F. Influence of condensation surface on solar distillation. Desalination 2013, 326, 37-45. [CrossRef]

17. Simsek, E.; Zhu, K.; Kashanchi, G.N.; Williams, M.J.; Galy, T.; Marszewski, M.; Tolbert, S.H.; Pilon, L. Light transfer through semi-transparent glass panes supporting pendant droplets. J. Quant. Spectrosc. Radiat. Transf. 2020, under review. [CrossRef]

18. Zhu, K.; Pilon, L. Transmittance of semitransparent windows with absorbing cap-shaped droplets condensed on their backside. J. Quant. Spectrosc. Radiat. Transf. 2017, 201, 53-63. [CrossRef]

19. Rubin, M. Optical properties of soda lime silica glasses. Sol. Energy Mater. 1985, 12, 275-288. [CrossRef]

20. Hale, G.M.; Querry, M.R. Optical constants of water in the 200-nm to 200- $\mu$ m wavelength region. Appl. Opt. 1973, 12, 555-563. [CrossRef]

21. Pottier, L.; Pruvost, J.; Deremetz, J.; Cornet, J.-F.; Legrand, J.; Dussap, C.-G. A fully predictive model for one-dimensional light attenuation by Chlamydomonas Reinhardtii A Torus Photobioreactor. Biotechnol. Bioeng. 2005, 91, 569-582. [CrossRef]

22. Lee, E.; Pruvost, J.; He, X.; Munipalli, R.; Pilon, L. Design tool and guidelines for outdoor photobioreactors. Chem. Eng. Sci. 2014, 106, 18-29. [CrossRef]

23. Cornet, J.-F.; Dussap, C.G.; Dubertret, G. A structured model for simulation of cultures of the cyanobacterium Spirulina Platensis Photobioreactors: I. Coupling between Light Transf. Growth Kinet. Biotechnol. Bioeng. 1992, 40, 817-825. [CrossRef] [PubMed]

24. Cornet, J.-F.; Dussap, C.G.; Gros, J.B.; Binois, C.; Lasseur, C. A simplified monodimensional approach for modeling coupling between radiant light transfer and growth kinetics in photobioreactors. Chem. Eng. Sci. 1995, 50, 1489-1500. [CrossRef]

25. Pruvost, J.; Cornet, J.-F.; Goetz, V.; Legrand, J. Theoretical investigation of biomass productivities achievable in solar rectangular photobioreactors for the cyanobacterium Arthrospira platensis. Biotechnol. Prog. 2012, 28, 699-714. [CrossRef] [PubMed]

26. Dunn, I.; Heinzle, E.; Ingham, J.; Prenosil, J. Biological Reaction Engineering: Dynamic Modelling Fundamentals with Simulation Examples, 2nd ed.; Wiley-VCH: Weinheim, Germany, 2003.

27. Takache, H.; Pruvost, J.; Cornet, J.-F. Kinetic modeling of the photosynthetic growth of Chlamydomonas Reinhardtii A Photobioreactor. Biotechnol. Prog. 2012, 28, 681-692. [CrossRef] [PubMed]

28. Versyck, K.J.; Claes, J.E.; van Impe, J.-F. Practical identification of unstructured growth kinetics by application of optimal experimental design. Biotechnol. Prog. 1997, 13, 524-531. [CrossRef]

29. Fouchard, S.; Pruvost, J.; Degrenne, B.; Titica, M.; Legrand, J. Kinetic modeling of light limitation and sulfur deprivation effects in the induction of hydrogen production with Chlamydomonas reinhardtii: Part I. Model Dev. Parameter Identification. Biotechnol. Bioeng. 2009, 102, 232-245. [CrossRef]

30. Cornet, J.-F.; Dussap, C.-G. A simple and reliable formula for assessment of maximum volumetric productivities in photobioreactors. Biotechnol. Prog. 2009, 25, 424-435. [CrossRef]

31. Pruvost, J.; Vooren, G.; Gouic, B.; Couzinet-Mossion, A.; Legrand, J. Systematic investigation of biomass and lipid productivity by microalgae in photobioreactors for biodiesel application. Bioresour. Technol. 2011, 2, 150-158. [CrossRef]

32. Slegers, P.M.; Wijffels, R.H.; van Straten, G.; van Boxtel, A.J.B. Design scenarios for flat panel photobioreactors. Appl. Energy 2011, 88, 3342-3353. [CrossRef]

33. Modest, M.F. Radiative Heat Transfer, 3rd ed.; Elsevier Inc.: Oxford, UK, 2013.

34. Howell, J.R.; Mengüç, M.P.; Siegel, R. Thermal Radiation Heat Transfer, 6th ed.; CRC Press: Taylor and Francis: Boca Raton, FL, USA, 2016.

35. Kandilian, R.; Pruvost, J.; Artu, A.; Lemasson, C.; Legrand, J.; Pilon, L. Comparison of experimentally and theoretically determined radiation characteristics of photosynthetic microorganisms. J. Quant. Spectrosc. Radiat. Transf. 2016, 175, 30-45. [CrossRef]

36. Gueymard, C. Simple Model of the Atmospheric Radiative Transfer of Sunshine (SMARTS). Version 2.9.5. 2005. Available online: https://www.nrel.gov/grid/solar-resource/smarts.html (accessed on 30 December 2020).

37. Figgis, B.; Nouviaire, A.; Wubulikasimu, Y.; Javed, W.; Guo, B.; Ait-Mokhtar, A.; Belarbi, R.; Ahzi, S.; Rémond, Y.; Ennaoui, A. Investigation of factors affecting condensation on soiled PV modules. Sol. Energy 2018, 159, 488-500. [CrossRef] 